\title{
Stationary isothermic surfaces in Euclidean 3-space *
}

\author{
Rolando Magnanini ${ }^{\dagger}$, Daniel Peralta-Salas ${ }^{\ddagger}$ and Shigeru Sakaguchi ${ }^{\S}$
}

March 2, 2018

\begin{abstract}
Let $\Omega$ be a domain in $\mathbb{R}^{3}$ with $\partial \Omega=\partial\left(\mathbb{R}^{3} \backslash \bar{\Omega}\right)$, where $\partial \Omega$ is unbounded and connected, and let $u$ be the solution of the Cauchy problem for the heat equation $\partial_{t} u=\Delta u$ over $\mathbb{R}^{3}$, where the initial data is the characteristic function of the set $\Omega^{c}=\mathbb{R}^{3} \backslash \Omega$. We show that, if there exists a stationary isothermic surface $\Gamma$ of $u$ with $\Gamma \cap \partial \Omega=\varnothing$, then both $\partial \Omega$ and $\Gamma$ must be either parallel planes or co-axial circular cylinders. This theorem completes the classification of stationary isothermic surfaces in the case that $\Gamma \cap \partial \Omega=\varnothing$ and $\partial \Omega$ is unbounded. To prove this result, we establish a similar theorem for uniformly dense domains in $\mathbb{R}^{3}$, a notion that was introduced by Magnanini, Prajapat \& Sakaguchi in MaPS. In the proof, we use methods from the theory of surfaces with constant mean curvature, combined with a careful analysis of certain asymptotic expansions and a surprising connection with the theory of transnormal functions.
\end{abstract}

Key words. heat equation, Cauchy problem, Euclidean 3-space, stationary isothermic surface, uniformly dense domain, transnormal function, constant mean curvature surface, plane, circular cylinder.

AMS subject classifications. Primary 35K05, 35K15; Secondary 53A10, 58J70.

${ }^{*}$ This research was partially supported by the ERC grant 335079 and the Spanish MINECO grant SEV-2011-0087, and by Grants-in-Aid for Scientific Research (B) ( $\sharp 20340031$ and $\sharp 26287020$ ) and for Challenging Exploratory Research $(\sharp 25610024)$ of Japan Society for the Promotion of Science. The first and third author have also been supported by the Gruppo Nazionale per l'Analisi Matematica, la Probabilità e le loro Applicazioni (GNAMPA) of the italian Istituto Nazionale di Alta Matematica (INdAM).

${ }^{\dagger}$ Dipartimento di Matematica U. Dini, Università di Firenze, viale Morgagni 67/A, 50134 Firenze, Italy. (magnanin@math.unifi.it).

${ }^{\ddagger}$ Instituto de Ciencias Matemáticas, Consejo Superior de Investigaciones Científicas, 28049 Madrid, Spain. (dperalta@icmat.es).

${ }^{\S}$ Research Center for Pure and Applied Mathematics, Graduate School of Information Sciences, Tohoku University, Sendai, 980-8579, Japan. (sigersak@m.tohoku.ac.jp). 


\section{Introduction}

Let $\Omega$ be a domain in $\mathbb{R}^{N}$ with $N \geq 2$. Consider the unique bounded solution $u=u(x, t)$ of the Cauchy problem:

$$
\partial_{t} u=\Delta u \text { in } \mathbb{R}^{N} \times(0,+\infty) \text { and } u=\mathcal{X}_{\Omega^{c}} \text { on } \mathbb{R}^{N} \times\{0\}
$$

where $\mathcal{X}_{\Omega^{c}}$ denotes the characteristic function of the set $\Omega^{c}=\mathbb{R}^{N} \backslash \Omega$. A hypersurface $\Gamma$ in $\mathbb{R}^{N}$ is called a stationary isothermic surface of $u$ if at each time $t$ the solution $u$ remains constant on $\Gamma$ (a constant depending on $t$ ). The following problem was raised in [MaPS]:

Classify all the domains $\Omega$ having a stationary isothermic surface.

For $N=2$ the answer is easy: $\partial \Omega$ is either a circle, a straight line or a couple of parallel straight lines (see [MaPS]). One can also easily show that, if $\partial \Omega$ is a sphere, a hyperplane or, up to rescalings, any spherical cylinder $\mathbb{S}^{k-1} \times \mathbb{R}^{N-k}, 2 \leq k \leq N-1$, then every level surface of $u$ is a stationary isothermic surface. Another interesting example is a helicoid $\mathcal{H}$ in $\mathbb{R}^{3}$. If $\Omega$ is a domain in $\mathbb{R}^{3}$ with $\partial \Omega=\mathcal{H}$, then $\mathcal{H}$ is a stationary isothermic surface of $u$ (see [MaPS, p. 4824]).

In order to study this problem, the notion of uniformly dense domains was introduced in [MaPS]. Let $B(x, r)$ be the open ball of positive radius $r$ and center $x \in \mathbb{R}^{N}$ and define the density

$$
\rho(x, r)=\frac{|\Omega \cap B(x, r)|}{|B(x, r)|},
$$

where $|\Omega \cap B(x, r)|$ and $|B(x, r)|$ denote the $N$-dimensional Lebesgue measure of the sets $\Omega \cap B(x, r)$ and $B(x, r)$, respectively. As defined in [MaPS], $\Omega$ is uniformly dense in the hypersurface $\Gamma$ if and only if there exists $r_{0} \in(\operatorname{dist}(\Gamma, \partial \Omega),+\infty]$ such that, for every fixed $r \in\left(0, r_{0}\right)$, the function $x \mapsto \rho(x, r)$ is constant on $\left.\Gamma\right]$ Thus, if $\partial \Omega=\partial\left(\mathbb{R}^{N} \backslash \bar{\Omega}\right)$ and $\Omega$ is uniformly dense in $\Gamma$, it is clear that any point $x \in \Gamma$ must have the same distance, say $R$, from $\partial \Omega$, i.e. $\Gamma$ and $\partial \Omega$ are parallel hypersurfaces.

In fact, stationary isothermic surfaces and uniformly dense domains are connected by the formula

$$
1-u(x, t)=2(4 \pi t)^{-N / 2} \int_{0}^{\infty}|\Omega \cap B(x, 2 \sqrt{t} s)| s e^{-s^{2}} d s
$$

that can be easily derived by applying the integration by parts formula and a change of variables to the representation formula (2.4) in [MaPS, page 4825]. Hence by the

\footnotetext{
${ }^{1}$ This assumption can be relaxed: it would be enough to assume that there exist two functions $r(x) \in$ $(\operatorname{dist}(\Gamma, \partial \Omega),+\infty)$ for $x \in \Gamma$ and $\rho_{0}(r) \geq 0$ for $r>0$ such that $\rho(x, r)=\rho_{0}(r)$ provided that $x \in \Gamma$ and $0<r<r(x)$.
} 
arguments used in [MaPS, Theorem 1.1], we see that $\Gamma$ is a stationary isothermic surface of $u$ if and only if $\Omega$ is uniformly dense in $\Gamma$ with $r_{0}=\infty$. Heuristically, this means that a stationary isothermic surface collects local and global information about the set $\Omega$, since that formula also informs us that the short and large time behavior of $u$ are respectively linked to the behavior of $\rho(x, r)$ for small and large values of $r$. The results presented in this paper only use the local information about $\Omega$, since they rely on the behavior of $\rho(x, r)$ for small values of $r$, that is when $r_{0}<\infty$. (Nevertheless, we believe that the assumption $r_{0}=\infty$, besides simplifying some arguments as in MaPS], is necessary to attempt a classification in general dimension of uniformly dense domains and, of course, stationary isothermic surfaces.)

The case, where $\Gamma \subset \partial \Omega$ and $\Omega$ is uniformly dense in $\Gamma$, is considered in MaPS, Theorems 1.2, 1.3, and 1.4]. In particular it is shown that, if $N=3$ and $\partial \Omega$ is connected, then $\partial \Omega$ must be a sphere, a circular cylinder, or a minimal surface. Also, if $\partial \Omega$ is a complete embedded minimal surface of finite total curvature in $\mathbb{R}^{3}$, then it must be a plane.

The case, where $\Gamma \cap \partial \Omega=\varnothing, \partial \Omega$ is bounded, and $\Omega$ is uniformly dense in $\Gamma$, is studied in [MaPS, Theorem 3.6] and [MaS3, Theorem 1.2], where in particular it is shown that $\partial \Omega$ must be a sphere, if it is connected. Here the boundedness of $\partial \Omega$ enables us to use Alexandrov's sphere theorem to reach the conclusion. The case, where $\Gamma \cap \partial \Omega=\varnothing, \partial \Omega$ is an entire graph over $\mathbb{R}^{N-1}$, and $\Gamma$ is a stationary isothermic surface of $u$, is analyzed in [MaS2, Theorem 2.3] and in [Sa, Theorem 2], and it is shown that $\partial \Omega$ must be a hyperplane under some additional conditions on $\partial \Omega$. In both of these cases the global conditions on $\partial \Omega$ play a key role to reach the conclusions. Therefore, in order to classify all the cases in which $\Gamma \cap \partial \Omega=\varnothing, \partial \Omega$ is unbounded, and $\Omega$ is uniformly dense in $\Gamma$ with no global assumptions on $\partial \Omega$, a new approach must be developed. In the present paper we show that such a classification is actually possible in $\mathbb{R}^{3}$.

To complete the picture, we mention that an interesting generalization of uniformly dense domains - the so called $K$-dense sets — is considered in [ABG], [MM1] and [MM2]: they correspond to the case in which the balls $B(x, r)=x+r B(0,1)$ in (1.2) are replaced by the family of sets $x+r K$, where $K$ is any fixed reference convex body. It is proved that, if $\Omega$ is a $K$-dense set for $\Gamma=\partial \Omega, 0<|\Omega|<\infty$ and $r_{0}=\infty$, then both $K$ and $\Omega$ must be ellipses (homothetic to one another) if $N=2$ ([ABG], [MM1]) and ellipsoids if $N \geq 3$ ([MM2]).

In the present paper, we work in $\mathbb{R}^{3}$ and complete the classification of unbounded 
stationary isothermic surfaces initiated in [MaPS], by the following theorem and corollary.

Theorem 1.1 Let $\Omega \subset \mathbb{R}^{3}$ be a domain with unbounded and connected boundary $\partial \Omega$ such that $\partial \Omega=\partial\left(\mathbb{R}^{3} \backslash \bar{\Omega}\right)$ and let $D$ be a domain with $\bar{D} \subset \Omega$. Consider a connected component $\Gamma$ of $\partial D$ satisfying

$$
\operatorname{dist}(\Gamma, \partial \Omega)=\operatorname{dist}(\partial D, \partial \Omega)
$$

and suppose that $D$ satisfies the interior cone condition on $\Gamma 2$

If $\Omega$ is uniformly dense in $\Gamma$, then $\partial \Omega$ and $\Gamma$ must be either parallel planes or co-axial circular cylinders.

Corollary 1.2 Let $\Omega, D$ and $\Gamma$ be as in Theorem 1.1. Assume that $\Gamma$ is a stationary isothermic surface of the solution $u$ of the Cauchy problem (1.1).

Then $\partial \Omega$ and $\Gamma$ must be either parallel planes or co-axial circular cylinders.

Recall that any point $x \in \Gamma$ has the same distance $R$ from $\partial \Omega$ if $\partial \Omega=\partial\left(\mathbb{R}^{3} \backslash \bar{\Omega}\right)$ and $\Omega$ is uniformly dense in $\Gamma$. By observing that

$$
\begin{aligned}
& 1-\rho(x, r)=\frac{\left|\Omega^{c} \cap B(x, r)\right|}{|B(x, r)|} \text { for every } r>0, \\
& \text { and } \frac{d}{d r}\left|\Omega^{c} \cap B(x, r)\right|=\left|\Omega^{c} \cap \partial B(x, r)\right| \text { for almost every } r>0,
\end{aligned}
$$

where $\left|\Omega^{c} \cap \partial B(x, r)\right|$ denotes the 2-dimensional Hausdorff measure of the set $\Omega^{c} \cap \partial B(x, r)$, we introduce another ratio:

$$
\sigma(x, r)=\frac{\left|\Omega^{c} \cap \partial B(x, r)\right|}{|\partial B(x, r)|} .
$$

Then, the proof of Theorem 1.1 relies on the observation that, being $\Omega$ uniformly dense in $\Gamma, \sigma(x, r)$ and all the coefficients of its asymptotic expansion for $r \rightarrow R+0$ are independent of $x$ if $x \in \Gamma$. The regularity in $r$ of $\sigma(x, r)$ descends from that of the uniformly dense domain $\Omega$, that we derive in Section 2 in general dimension and under the assumption that $\partial \Omega=\partial\left(\mathbb{R}^{N} \backslash \bar{\Omega}\right)$. The computation of the first coefficient of the asymptotic expansion of $\sigma(x, r)$ for $r \rightarrow R+0$ was already carried out in [MaPS] for any $N$, while that of the second one is performed for $N=3$ in Proposition 3.1. which is the most technical part of the paper (in the Appendix, we collect the calculations of some definite integrals needed in its proof).

\footnotetext{
${ }^{2}$ This assumption can be replaced by requiring that $\Gamma$ satisfies the following center of mass condition: for any $x \in \Gamma$, there is a number $r \in\left(0, r_{0}\right)$ such that $x$ is not the center of mass of $\Omega^{c} \cap B(x, r)$.
} 
A key role is played by Propositions 2.3 and 3.3 that give useful geometrical insight about the first and second coefficients of the aforementioned asymptotic expansion. In summary, if $\Omega$ is uniformly dense in $\Gamma$, those propositions imply the existence of an intermediate surface $\Gamma_{*}$ between $\partial \Omega$ and $\Gamma$, parallel to both $\partial \Omega$ and $\Gamma$, that has constant mean curvature $H^{*}$ (Proposition 2.3) and of a polynomial $\Psi=\Psi(t)$ of degree 4 at most such that

$$
\left\|\nabla K^{*}\right\|^{2}=\Psi\left(K^{*}\right) \text { on } \Gamma_{*}
$$

(see Proposition 3.3 and the arguments yielding (4.2) in Section 4). Here, $\left\|\nabla K^{*}\right\|$ is the length of the gradient (with respect to the induced metric of $\Gamma_{*}$ ) of the Gauss curvature $K^{*}$ of $\Gamma_{*}$. In particular, (1.6) tells us that $K^{*}$ is a transnormal function if $K^{*}$ is not constant(see [W], $\mathrm{Mi}],[\mathrm{B}]$ ).

The proof of our classification of uniformly dense sets and stationary isothermic surfaces - Theorem 1.1 and Corollary 1.2 - is in Section 4. We obtain it in two ways: by combining ideas from the theories of minimal surfaces and surfaces with constant mean curvature properly embedded in $\mathbb{R}^{3}$, and the theory of transnormal functions; by directly checking that the Gauss curvature of catenoids, helicoids and unduloids (that, together with planes and circular cylinders, are the only surfaces of constant mean curvature that we need to consider, as we will show) does not satisfy Eq. (1.6) with a polynomial function $\Psi$.

Finally, in Section 5 we present a generalization of [MaPS, Theorem 1.4, p. 4824] by using the theory of properly embedded minimal surfaces of finite topology in $\mathbb{R}^{3}$.

\section{Regularity of uniformly dense sets}

For later use, we introduce some notations and recall some well-known facts. We define the parallel surface

$$
\Gamma_{\rho}=\{x \in \Omega: \operatorname{dist}(x, \partial \Omega)=\rho\} \quad \text { for } \quad 0<\rho<R .
$$

Also, $\nu$ and $\kappa_{1}, \cdots, \kappa_{N-1}$ will denote the inward unit normal vector to $\partial \Omega$ and the principal curvatures of $\partial \Omega$ with respect to $\nu$ at a point $\xi \in \partial \Omega$. For notational simplicity, the explicit dependence of these quantities on the point $\xi$ will be indicated only when it is needed to avoid ambiguities. However, be aware that $\hat{\nu}$ and $\hat{\kappa}_{1}, \cdots, \hat{\kappa}_{N-1}$ will denote the outward unit normal vector to $\partial D$ on $\Gamma$ and the principal curvatures of $\Gamma$ with respect to $\hat{\nu}$ at the point $x=\xi+R \nu \in \Gamma$. 
In the spirit of [MaS3, Lemma 3.1] and by the arguments used in MaPS, the proofs of Theorems 2.4 and 2.5], we obtain the following lemma that is partially motivated by Remark 2.2 below.

Lemma 2.1 Let $\Omega$ be a domain in $\mathbb{R}^{N}$ with $N \geq 2$ and $\partial \Omega=\partial\left(\mathbb{R}^{N} \backslash \bar{\Omega}\right)$, and let $D$ be a domain in $\mathbb{R}^{N}$ with $\bar{D} \subset \Omega$. Consider a connected component $\Gamma$ of $\partial D$ satisfying (1.4) and suppose that $D$ satisfies the interior cone condition on $\Gamma$.

If $\Omega$ is uniformly dense in $\Gamma$, then the following properties hold:

(1) There exists a number $R>0$ such that $\operatorname{dist}(x, \partial \Omega)=R$ for every $x \in \Gamma$;

(2) $\Gamma$ is a real analytic hypersurface embedded in $\mathbb{R}^{N}$;

(3) there exists a connected component $\gamma$ of $\partial \Omega$, which is also a real analytic hypersurface embedded in $\mathbb{R}^{N}$, such that the mapping $\gamma \ni \xi \mapsto x(\xi) \equiv \xi+R \nu(\xi) \in \Gamma$ is a diffeomorphism; in particular, $\gamma$ and $\Gamma$ are parallel hypersurfaces at distance $R$;

(4) it holds that

$$
\kappa_{j}<\frac{1}{R} \text { on } \gamma, \text { for every } j=1, \ldots, N-1 \text {; }
$$

(5) there exists a number $c>0$ such that

$$
\prod_{j=1}^{N-1}\left(1-R \kappa_{j}\right)=c \text { on } \gamma .
$$

Proof. Since $\Omega$ is uniformly dense in $\Gamma$, there exists $r_{0} \in(\operatorname{dist}(\Gamma, \partial \Omega),+\infty]$ such that for every fixed $r \in\left(0, r_{0}\right)$ the function $x \mapsto\left|\Omega^{c} \cap B(x, r)\right|$ is also constant on $\Gamma$. Therefore, property (1) holds for some $R>0$, since $\partial \Omega=\partial\left(\mathbb{R}^{N} \backslash \bar{\Omega}\right)$ and $\Gamma \cap \partial \Omega=\varnothing$. Moreover Eq. (1.4) yields that $R=\operatorname{dist}(\Gamma, \partial \Omega)=\operatorname{dist}(\partial D, \partial \Omega)$.

First, let us show that $\Gamma$ is a $C^{\infty}$ hypersurface. Take an arbitrary function $\eta \in$ $C_{0}^{\infty}\left(0, r_{0}\right)$ and set $\psi(x)=\eta(|x|)$ for $x \in \mathbb{R}^{N}$. Then $\psi \in C_{0}^{\infty}\left(\mathbb{R}^{N}\right)$, supp $(\psi) \subset B\left(0, r_{0}\right)$ and the convolution $\psi \star \mathcal{X}_{\Omega^{c}}$ belongs to $C^{\infty}\left(\mathbb{R}^{N}\right)$. Moreover we have that

$$
\psi \star \mathcal{X}_{\Omega^{c}}(x)=\int_{\Omega^{c} \cap B\left(x, r_{0}\right)} \eta(|x-y|) d y=\int_{0}^{r_{0}} \eta(r)\left|\Omega^{c} \cap \partial B(x, r)\right| d r,
$$

where $\left|\Omega^{c} \cap \partial B(x, r)\right|$ denotes the $(N-1)$-dimensional Hausdorff measure of the set $\Omega^{c} \cap$ $\partial B(x, r)$. 
The function $\psi \star \mathcal{X}_{\Omega^{c}}$ is constant on $\Gamma$. In fact, if we fix two points $p, q \in \Gamma$ arbitrarily, being $\Omega$ uniformly dense in $\Gamma$, we have that

$$
\left|\Omega^{c} \cap B(p, r)\right|=\left|\Omega^{c} \cap B(q, r)\right| \text { for every } r \in\left(0, r_{0}\right)
$$

and hence, by differentiating with respect to $r$ both sides, that

$$
\left|\Omega^{c} \cap \partial B(p, r)\right|=\left|\Omega^{c} \cap \partial B(q, r)\right| \text { for almost every } r \in\left(0, r_{0}\right) .
$$

Thus, if we show that for every $x \in \Gamma$ there exists a function $\eta \in C_{0}^{\infty}\left(0, r_{0}\right)$ such that $\nabla\left(\psi \star \mathcal{X}_{\Omega^{c}}\right)(x) \neq 0$, then we can conclude that $\Gamma$ is a $C^{\infty}$ hypersurface, by the implicit function theorem. Suppose that there exists a point $x_{0} \in \Gamma$ such that

$$
\nabla\left(\psi \star \mathcal{X}_{\Omega^{c}}\right)\left(x_{0}\right)=0 \text { for every } \eta \in C_{0}^{\infty}\left(0, r_{0}\right)
$$

it follows that

$$
\int_{0}^{r_{0}}\left[\int_{\Omega^{c} \cap \partial B\left(x_{0}, r\right)}\left(x_{0}-y\right) d S_{y}\right] \frac{\eta^{\prime}(r)}{r} d r=0 \text { for every } \eta \in C_{0}^{\infty}\left(0, r_{0}\right),
$$

where $d S_{y}$ denotes the area element of the sphere $\partial B\left(x_{0}, r\right)$. This, together with the fact that $\Omega^{c} \cap \partial B\left(x_{0}, r\right)=\varnothing$ for $0<r<R$, gives that the surface integral in the brackets is zero for almost every $r \in\left(0, r_{0}\right)$, and hence that

$$
\int_{\Omega^{c} \cap B\left(x_{0}, r\right)}\left(x_{0}-y\right) d y=0 \text { for every } r \in\left(0, r_{0}\right)
$$

- that is, $x_{0}$ must be the center of mass of $\Omega^{c} \cap B\left(x_{0}, r\right)$ for every $r \in\left(0, r_{0}\right)$.

By the same argument as in [MaPS, the proof of Theorem 2.5], the interior cone condition for $\Gamma$ gives a contradiction. Thus, $\Gamma$ is a $C^{\infty}$ hypersurface embedded in $\mathbb{R}^{N} 3$

Now, since $\Gamma$ is a connected component of $\partial D$, we notice that, in view of (1.4), property (1) and the smoothness of $\Gamma$ imply that

$$
\text { for each } x \in \Gamma \text { there exists a unique } \xi \in \partial \Omega \text { satisfying } x \in \partial B(\xi, R) \text {, }
$$

since $\xi-x$ must be parallel to $\hat{\nu}(x)$. Note that $\xi=x+R \hat{\nu}(x)$, and in view of property (1) and (2.4), comparing the principal curvatures at $x$ of $\Gamma$ with those of the sphere $\partial B(\xi, R)$ yields that

$$
\hat{\kappa}_{j} \leq \frac{1}{R} \text { on } \Gamma \text {, for every } j=1, \ldots, N-1 .
$$

\footnotetext{
${ }^{3}$ It is clear that the following assumption would suffice: $\Gamma$ is an immersed topological surface satisfying the center of mass condition, as defined in the previous footnote.
} 
Since $\Gamma$ is a connected component of $\partial D$, then $\Gamma$ is oriented and divides $\mathbb{R}^{N}$ into two domains. Let $E$ be the one of them which does not intersect $D$. By property (1) and (1.4), $E \cap\left(\mathbb{R}^{N} \backslash \bar{\Omega}\right)$ contains a point, say, $z$. Set $R_{0}=\operatorname{dist}(z, \Gamma)$. Then $R_{0}>R$ and there exists a point $p_{0} \in \Gamma$ such that $R_{0}=\left|z-p_{0}\right|$. Comparing the principal curvatures at $p_{0}$ of $\Gamma$ with those of the sphere $\partial B\left(z, R_{0}\right)$, yields that $\hat{\kappa}_{j}\left(p_{0}\right) \leq \frac{1}{R_{0}}<\frac{1}{R}$ for every $j=1, \ldots, N-1$. By continuity, there exists a small $\delta_{0}>0$ such that

$$
\hat{\kappa}_{j}(x)<\frac{1}{R} \quad \text { for every } \quad x \in \Gamma \cap \overline{B\left(p_{0}, \delta_{0}\right)} \text { and every } j=1, \ldots, N-1,
$$

and the mapping $\Gamma \cap B\left(p_{0}, \delta_{0}\right) \ni x \mapsto \xi(x) \equiv x+R \hat{\nu}(x) \in \partial \Omega$ is a diffeomorphism onto its image $\gamma_{0}$ given by

$$
\gamma_{0}=\xi\left(\Gamma \cap B\left(p_{0}, \delta_{0}\right)\right)(\subset \partial \Omega)
$$

Hence $\gamma_{0}$ is a portion of a $C^{\infty}$ hypersurface, since $\Gamma$ is a $C^{\infty}$ hypersurface.

Notice that the principal curvatures $\kappa_{1}, \cdots, \kappa_{N-1}$ of $\gamma_{0}$ satisfy

$$
-\kappa_{j}(\xi(x))=\frac{\hat{\kappa}_{j}(x)}{1-R \hat{\kappa}_{j}(x)} \text { for every } x \in \Gamma \cap B\left(p_{0}, \delta_{0}\right) \text { and every } j=1, \ldots, N-1 .
$$

Therefore, since $1-R \kappa_{j}(\xi(x))=1 /\left(1-R \hat{\kappa}_{j}(x)\right)$, we see that (2.6) is equivalent to

$$
\kappa_{j}<\frac{1}{R} \text { on } \gamma_{0} \text { for every } j=1, \ldots, N-1 \text {. }
$$

Here, notice that $\partial \Omega$ may have a point of selfcontact, since we only assume that $\partial \Omega=\partial\left(\mathbb{R}^{N} \backslash \bar{\Omega}\right)$. For this reason set

$$
\gamma_{0}^{*}=\left\{\xi \in \gamma_{0}: \xi \text { is a point of selfcontact of } \partial \Omega\right\} .
$$

Then $\gamma_{0}^{*}$ does not contain any interior points in $\gamma_{0}$, since $\gamma_{0}$ is a portion of a $C^{\infty}$ hypersurface and $\partial \Omega=\partial\left(\mathbb{R}^{N} \backslash \bar{\Omega}\right)$.

Let $P, Q \in \gamma_{0} \backslash \gamma_{0}^{*}$ be any two points and set $\xi(p)=P, \xi(q)=Q$ for $p, q \in \Gamma \cap B\left(p_{0}, \delta_{0}\right)$. Then, it follows from (2.3) and the smoothness of $\gamma_{0}$ that there exists a small number $\varepsilon>0$ satisfying

$$
\left|\Omega^{c} \cap \partial B(p, r)\right|=\left|\Omega^{c} \cap \partial B(q, r)\right| \text { for every } r \in(R, R+\varepsilon) .
$$

Hence we can use [MaPS, Theorem 5.5], with $\mathbb{R}^{N} \backslash \bar{\Omega}$ in place of $\Omega$, to get

$$
\left[\prod_{j=1}^{N-1}\left(1-R \kappa_{j}(P)\right)\right]^{-\frac{1}{2}}=\left[\prod_{j=1}^{N-1}\left(1-R \kappa_{j}(Q)\right)\right]^{-\frac{1}{2}}
$$


Therefore, since $\gamma_{0}^{*}$ does not contain any interior points in $\gamma_{0}$, by continuity we conclude that

$$
\prod_{j=1}^{N-1}\left(1-R \kappa_{j}\right)=c \text { on } \gamma_{0}
$$

where, for instance, $c$ is the (positive) value of the right-hand side of (2.9) at the point $P_{0}=\xi\left(p_{0}\right) \in \gamma_{0}$. Since $1-R \kappa_{j}(\xi(x))=1 /\left(1-R \hat{\kappa}_{j}(x)\right)$, we see that

$$
\prod_{j=1}^{N-1}\left[1-R \hat{\kappa}_{j}(x)\right]=c^{-1} \text { for every } x \in \Gamma \cap B\left(p_{0}, \delta_{0}\right) .
$$

Define a set $J \subset \Gamma$ by

$$
J=\left\{p \in \Gamma: \max _{1 \leq j \leq N-1} \hat{\kappa}_{j}(p)<\frac{1}{R} \text { and } \prod_{j=1}^{N-1}\left[1-R \hat{\kappa}_{j}(p)\right]=c^{-1}\right\} .
$$

By the previous argument we notice that $J$ is a relatively open subset of $\Gamma$ and $J \neq \varnothing$. Moreover, $J$ is a relatively closed subset of $\Gamma$. Indeed, for any sequence of points $p_{k} \in J$ converging to some $p \in \Gamma$ as $k \rightarrow \infty$, in the limit we would get that

$$
\max _{1 \leq j \leq N-1} \hat{\kappa}_{j}(p) \leq \frac{1}{R} \text { and } \prod_{j=1}^{N-1}\left[1-R \hat{\kappa}_{j}(p)\right]=c^{-1}(>0),
$$

and the second equality implies that the first inequality must be strict; thus, $J$ is closed. Since $\Gamma$ is connected, we conclude that $J=\Gamma$. Also, the regularity theory for nonlinear elliptic equations implies that $\Gamma$ is a real analytic hypersurface, since $\Gamma$ is locally a graph of a function which satisfies a Monge-Ampère type equation coming from the second equality of (2.10). Let us set

$$
\gamma=\left\{\xi(x) \in \mathbb{R}^{N}: x \in \Gamma\right\}
$$

Then $\gamma$ does not have any points of selfcontact, that is, the mapping $\xi: \Gamma \rightarrow \partial \Omega$ is injective. Indeed, suppose that there exists a point of selfcontact $P_{*} \in \gamma$, that is, there exist two open portions $\gamma_{+}, \gamma_{-}$of the manifold $\gamma$ containing a common point $P_{*}$. Hence we have two points $p_{+}, p_{-} \in \Gamma$ and two inward normal vectors $\nu^{+}$and $\nu^{-}$at $P_{*} \in \gamma(\subset \partial \Omega)$ satisfying

$$
\nu^{+}+\nu^{-}=0, p_{+}=P_{*}+R \nu^{+}, \text {and } p_{-}=P_{*}+R \nu^{-} .
$$

Denote by $\kappa_{1}^{ \pm}, \cdots, \kappa_{N-1}^{ \pm}$the principal curvatures of $\gamma_{ \pm}$at $P_{*} \in \gamma$ with respect to the inward unit normal vectors $\nu^{ \pm}$to $\partial \Omega$, respectively. Then we observe that

$$
-\frac{1}{R}<\kappa_{j}^{ \pm}<\frac{1}{R} \text { for every } j=1, \ldots, N-1 .
$$


since $J=\Gamma$ and $P_{*}$ is the point of selfcontact of $\gamma$. As before, take a point $Q \in \gamma$ which is not a point of selfcontact and set $\xi(q)=Q$ for $q \in \Gamma$. Then it follows from (2.3) and the smoothness of $\gamma$ that there exists a small number $\varepsilon_{0}>0$ satisfying

$$
\left|\Omega^{c} \cap \partial B\left(p_{+}, r\right)\right|=\left|\Omega^{c} \cap \partial B(q, r)\right| \text { for every } r \in\left(R, R+\varepsilon_{0}\right) .
$$

Hence, in view of (2.12) and (2.13), we can use [MaPS, Theorem 5.5] again to get

$$
\left[\prod_{j=1}^{N-1}\left(1-R \kappa_{j}^{+}\right)\right]^{-\frac{1}{2}}-\left[\prod_{j=1}^{N-1}\left(1+R \kappa_{j}^{-}\right)\right]^{-\frac{1}{2}}=\left[\prod_{j=1}^{N-1}\left(1-R \kappa_{j}(Q)\right)\right]^{-\frac{1}{2}} .
$$

This is a contradiction, since (2.9) in which $\gamma_{0}$ is replaced by $\gamma$ holds true from the fact that $J=\Gamma$.

Therefore, since $\hat{\kappa}_{j}<1 / R$ on $\Gamma$ for every $j=1, \ldots, N-1$, we see that the injective mapping $\Gamma \ni x \mapsto \xi(x) \equiv x+R \hat{\nu}(x) \in \gamma$ is a real analytic diffeomorphism because of the analyticity of $\Gamma$, and $\gamma$ is a real analytic hypersurface embedded in $\mathbb{R}^{N}$ which is a connected component of $\partial \Omega$. Since the mapping: $\gamma \ni \xi \mapsto x(\xi) \equiv \xi+R \nu(\xi) \in \Gamma$ is the inverse mapping of the previous diffeomorphism, property (3) holds. Both properties (4) and (5) follow from the fact that $J=\Gamma$. The proof is complete.

Remark 2.2 In [MaS3, Lemma 3.1 and its proof, pp. 2026-2029], the first and third authors of this paper did not take care of the case in which $\gamma$ has points of self-contact. Thus, Lemma 2.1 completes the proof of [MaS3, Lemma 3.1, p. 2026] for the case of the Cauchy problem.

Still, the argument we used to obtain (2.14) does not work in the case of the initialboundary value problem for the heat equation with boundary value 1 and initial value 0 - the matzoh ball soup setting considered initially in [MaS1]. Hence, statement 3 of [MaS3, Lemma 3.1, p. 2026] should be corrected in such a way that $\gamma$ is an immersed hypersurface in $\mathbb{R}^{N}$. Then $\gamma$ may have points of self-contact.

On the contrary, the reflection argument due to Alexandrov works for a bounded domain $\Omega$, even if $\partial \Omega$ contains points of self-contact (see $[\mathrm{A}]$ ). So the statement of Remark right after [MaS3, Lemma 3.1, p. 2026] still holds true.

The following proposition follows directly from Lemma 2.1 and is one of the key ingredients in the proof of Theorem 1.1. We preliminarily notice that, under the assumptions of Theorem 1.1, there exists $R>0$ such that

$$
\operatorname{dist}(x, \partial \Omega)=R \text { for every } x \in \Gamma,
$$


since $\partial \Omega=\partial\left(\mathbb{R}^{3} \backslash \bar{\Omega}\right)$ and $\Omega$ is uniformly dense in $\Gamma$. Also, since $\partial \Omega$ is connected, Lemma 2.1 and (2.15) imply that

$$
\gamma=\partial \Omega, \text { and } \partial \Omega \text { and } \Gamma \text { are parallel surfaces at distance } R>0 .
$$

Furthermore, both $\partial \Omega$ and $\Gamma$ are embedded in $\mathbb{R}^{3}$.

Proposition 2.3 Under the assumptions of Theorem 1.1, set $\rho_{*}=R /(1+\sqrt{c})$, where $c>0$ is the number in (2.2) in Lemma 2.1, and

$$
\Gamma_{*}=\left\{x \in \Omega: \operatorname{dist}(x, \partial \Omega)=\rho_{*}\right\} .
$$

Then, $\Gamma_{*}$ is a real analytic hypersurface parallel to $\partial \Omega, \Gamma_{*}$ is embedded in $\mathbb{R}^{3}$, and $\Gamma_{*}$ has a constant mean curvature

$$
H^{*}=\frac{1-c}{2 R \sqrt{c}},
$$

where the normal to $\Gamma_{*}$ is chosen to point in the same direction as the inward normal to $\partial \Omega$. In particular, $\Gamma_{*}$ is a properly embedded surface with constant mean curvature (or a properly embedded minimal surface when $c=1$ ) in $\mathbb{R}^{3}$, and hence it is complete.

Proof. Since both $\partial \Omega$ and $\Gamma$ are embedded in $\mathbb{R}^{3}$ and the mapping $\partial \Omega \ni \xi \mapsto \xi+\rho_{*} \nu(\xi) \in \Gamma_{*}$ is a diffeomorphism because $0<\rho_{*}<R$, we see that $\Gamma_{*}$ is also a real analytic hypersurface embedded in $\mathbb{R}^{3}$ and $\Gamma_{*}$ is parallel to both $\partial \Omega$ and $\Gamma$.

For $0<\rho<R$, we denote by $\kappa_{1}^{\rho}$ and $\kappa_{2}^{\rho}$ the principal curvatures of $\Gamma_{\rho}$ at $x=\xi+\rho \nu(\xi) \in$ $\Gamma_{\rho}$ with respect to the unit normal vector to $\Gamma_{*}$ with the same direction as $\nu(\xi)$. Then

$$
\kappa_{j}(\xi)=\frac{\kappa_{j}^{\rho}(x)}{1+\rho \kappa_{j}^{\rho}(x)}(j=1,2) \text { for every } \xi \in \partial \Omega .
$$

Substituting these in Eq. (2.2) yields

$$
c\left(1+\rho \kappa_{1}^{\rho}\right)\left(1+\rho \kappa_{2}^{\rho}\right)=\left[1+(\rho-R) \kappa_{1}^{\rho}\right]\left[1+(\rho-R) \kappa_{2}^{\rho}\right] .
$$

Hence, by letting $\rho=\rho_{*}$, with $\rho_{*}=R /(1+\sqrt{c})$, and $\Gamma_{*}=\Gamma_{\rho_{*}}$, we see that

$$
H^{*}=\frac{\kappa_{1}^{\rho_{*}}+\kappa_{2}^{\rho_{*}}}{2}=\frac{1-c}{2 R \sqrt{c}} \text {. }
$$

Let us see that $\Gamma_{*}$ is properly embedded in $\mathbb{R}^{3}$. Observe that $\Gamma_{*}=\{x \in \bar{\Omega}$ : $\left.\operatorname{dist}(x, \partial \Omega)=\rho_{*}\right\}$ where $\Omega$ is replaced by $\bar{\Omega}$. Since the $\operatorname{distance}$ function $\operatorname{dist}(x, \partial \Omega)$ is continuous on $\mathbb{R}^{3}$, we see that $\Gamma_{*}$ is closed in $\mathbb{R}^{3}$. Let $K$ be an arbitrary compact subset of $\mathbb{R}^{3}$. Then $\Gamma_{*} \cap K$ is also compact in $\mathbb{R}^{3}$. Let $\left\{p_{n}\right\}$ be an arbitrary sequence in 
$\Gamma_{*} \cap K$. By the Bolzano -Weierstraß theorem, $\left\{p_{n}\right\}$ has a convergent subsequence in $\mathbb{R}^{3}$. Let $p \in \Gamma_{*} \cap K$ be its limit point. Since $p \in \Gamma_{*}$, the smoothness of $\Gamma_{*}$ yields that there exists $\delta>0$ such that $B(p, \delta) \cap \Gamma_{*}$ is represented by a real analytic graph over the tangent plane of $\Gamma_{*}$ at $p$. This shows that the above subsequence also converges to $p$ with respect to the induced metric of $\Gamma_{*}$, which means that $\Gamma_{*}$ is properly embedded in $\mathbb{R}^{3}$. (Similarly, both $\partial \Omega$ and $\Gamma$ are properly embedded in $\mathbb{R}^{3}$.)

\section{Asymptotic expansions for $\sigma(x, r)$}

The second key ingredient in the proof of Theorem 1.1 is Proposition 3.1 below, in which we prove an asymptotic formula for $4 \pi \cdot \sigma(x, R+s)\left(=\left|\partial B(x, R+s) \cap \Omega^{c}\right| /(R+s)^{2}\right)$ as $s \rightarrow+0$, where $R>0$ is given in (2.15) and $\sigma(x, r)$ is defined in (1.5). The ensuing Proposition 3.3 will then clarify the geometric meaning of the function $g$ in (3.1). In Proposition 3.1, we choose a principal coordinate system $z=\left(z_{1}, z_{2}, z_{3}\right)$ with the origin at $\xi \in \partial \Omega$ and such that, in some neighborhood of $\xi, \partial \Omega$ is represented by the graph $z_{3}=\varphi\left(z_{1}, z_{2}\right)$, with the $z_{3}$ coordinate axis lying in the direction $-\nu(\xi)$ and

$$
\varphi\left(z_{1}, z_{2}\right)=-\frac{1}{2} \kappa_{1}(\xi) z_{1}^{2}-\frac{1}{2} \kappa_{2}(\xi) z_{2}^{2}+O\left(\left(z_{1}^{2}+z_{2}^{2}\right)^{\frac{3}{2}}\right) \quad \text { as } \sqrt{z_{1}^{2}+z_{2}^{2}} \rightarrow 0 .
$$

Hereafter, we abbreviate the partial derivatives of $\varphi$ with respect to $z_{1}$ and $z_{2}$ by subscripts:

$$
\varphi_{1}=\frac{\partial \varphi}{\partial z_{1}}, \varphi_{11}=\frac{\partial^{2} \varphi}{\partial z_{1}^{2}}, \varphi_{112}=\frac{\partial^{3} \varphi}{\partial z_{2} \partial z_{1}^{2}} \text { and so on. }
$$

Proposition 3.1 Let $\xi \in \partial \Omega$ and set $x=\xi+R \nu(\xi) \in \Gamma$. Under the assumptions of Theorem 1.1, we have:

$$
\frac{\left|\partial B(x, R+s) \cap \Omega^{c}\right|}{(R+s)^{2}}=\frac{2 \pi}{\sqrt{c}} \frac{s}{R}+\frac{\pi}{8 c \sqrt{c}}[h(K)+g]\left(\frac{s}{R}\right)^{2}+O\left(s^{\frac{5}{2}}\right) \quad \text { as } s \downarrow 0 .
$$

Here, $K=\kappa_{1}(\xi) \kappa_{2}(\xi)$ is the Gauss curvature of the surface $\partial \Omega$ at the point $\xi$ and $h$ is a 2-degree polynomial:

$$
h(t)=\left(R^{2} t+c-1\right)^{2}-4 c(c+3) .
$$

Moreover, $g \leq 0$ on $\partial \Omega$ and $g=0$ if and only if the third-order derivatives $\varphi_{111}$ and $\varphi_{222}$ of the function $\varphi$ defined above vanish at the origin.

The starting point of the proof of this proposition is Lemma 3.2, for which we need ad hoc notations and settings, in the spirit of those introduced in [MaPS]. 
In fact, we shall use the principal coordinate directions introduced before the statement of Proposition 3.1 without further mention. Also, for sufficiently small $s>0$, each point $w \in \partial B(x, R+s) \cap \Omega^{c}$ can be parameterized by a spherical coordinate system with the origin at $x \in \Gamma$ as

$$
w=x+(R+s)(\sin \eta \cos \theta, \sin \eta \sin \theta, \cos \eta), 0 \leq \eta \leq \eta(s, \theta), 0 \leq \theta<2 \pi,
$$

where $\eta=\eta(s, \theta)(0 \leq \theta \leq 2 \pi)$ represents the closed curve $\partial B(x, R+s) \cap \partial \Omega$ in that system. Notice that, for sufficiently small $s>0, \eta=\eta(s, \theta)$ satisfies

$$
G(\eta, s, \theta)=0 \text { for every } 0 \leq \theta \leq 2 \pi
$$

where the function $G=G(\eta, s, \theta)$ is given by

$$
G(\eta, s, \theta)=(R+s) \cos \eta-R-\varphi((R+s) \sin \eta \cos \theta,(R+s) \sin \eta \sin \theta) .
$$

Thus, we obtain:

$$
\frac{\left|\partial B(x, R+s) \cap \Omega^{c}\right|}{(R+s)^{2}}=\int_{0}^{2 \pi} d \theta \int_{0}^{\eta(s, \theta)} \sin \eta d \eta=\int_{0}^{2 \pi}(1-\cos \eta(s, \theta)) d \theta .
$$

Lemma 3.2 There exists a sequence $\left\{b_{j}(\theta)\right\}_{j=1}^{\infty}$ such that $b_{1}>0$ and $\eta=\eta(s, \theta)$ is expanded as the Puiseux series in s:

$$
\eta(s, \theta)=\sum_{j=1}^{\infty} b_{j}(\theta) s^{\frac{j}{2}} \text { for small } s \geq 0,
$$

and as $s \downarrow 0$

$$
\begin{aligned}
\frac{\left|\partial B(x, R+s) \cap \Omega^{c}\right|}{(R+s)^{2}}= & \frac{1}{2} \int_{0}^{2 \pi} b_{1}^{2} d \theta s+\int_{0}^{2 \pi} b_{1} b_{2} d \theta s^{\frac{3}{2}} \\
& +\frac{1}{2} \int_{0}^{2 \pi}\left(b_{2}^{2}+2 b_{1} b_{3}-\frac{1}{12} b_{1}^{4}\right) d \theta s^{2}+O\left(s^{\frac{5}{2}}\right) .
\end{aligned}
$$

Proof. Since the function $G$ given by (3.4) satisfies

$$
G(0,0, \theta)=0 \text { and } \frac{\partial G}{\partial s}(0,0, \theta)=1 \text { for every } 0 \leq \theta \leq 2 \pi
$$

by the implicit function theorem there exists a sequence $\left\{a_{j}(\theta)\right\}_{j=1}^{\infty}$ such that $s=s(\eta, \theta)$ is written as

$$
s=\sum_{j=1}^{\infty} a_{j}(\theta) \eta^{j} \text { for small } \eta \geq 0 .
$$


By differentiating the identity $G(\eta, s(\eta, \theta), \theta)=0$ with respect to $\eta$, we get

$$
\begin{aligned}
0= & s_{\eta} \cos \eta+(R+s)(-\sin \eta) \\
& -\varphi_{1}((R+s) \sin \eta \cos \theta,(R+s) \sin \eta \sin \theta)\left((R+s) \cos \eta \cos \theta+s_{\eta} \sin \eta \cos \theta\right) \\
& -\varphi_{2}((R+s) \sin \eta \cos \theta,(R+s) \sin \eta \sin \theta)\left((R+s) \cos \eta \sin \theta+s_{\eta} \sin \eta \sin \theta\right) .
\end{aligned}
$$

By setting $\eta=0$, we get

$$
a_{1}(\theta)=s_{\eta}(0, \theta)=0 .
$$

Differentiating the above identity with respect to $\eta$ once more and putting $\eta=0$ yield that

$$
0=s_{\eta \eta}(0, \theta)-R+R^{2} \kappa_{1}(\xi) \cos ^{2} \theta+R^{2} \kappa_{2}(\xi) \sin ^{2} \theta
$$

and hence

$$
\begin{aligned}
a_{2}(\theta) & =\frac{1}{2} s_{\eta \eta}(0, \theta)=\frac{1}{2} R\left[\left(1-R \kappa_{1}(\xi)\right) \cos ^{2} \theta+\left(1-R \kappa_{2}(\xi)\right) \sin ^{2} \theta\right] \\
& \geq \frac{1}{2} R\left[1-R \max \left\{\kappa_{1}(\xi), \kappa_{1}(\xi)\right\}\right]>0 .
\end{aligned}
$$

In view of (3.8), (3.9), and (3.10), we see that there exists a sequence $\left\{b_{j}(\theta)\right\}_{j=1}^{\infty}$ such that $b_{1}>0$ and $\eta=\eta(s, \theta)$ is expanded as the Puiseux series (3.6) in $s$. With the aid of (3.6), we calculate for $\eta=\eta(s, \theta)$

$$
\begin{aligned}
1-\cos \eta & =\frac{1}{2} \eta^{2}-\frac{1}{24} \eta^{4}+O\left(\eta^{6}\right) \\
& =\frac{1}{2} b_{1}^{2} s+b_{1} b_{2} s^{\frac{3}{2}}+\frac{1}{2}\left(b_{2}^{2}+2 b_{1} b_{3}-\frac{1}{12} b_{1}^{4}\right) s^{2}+O\left(s^{\frac{5}{2}}\right) \quad \text { as } s \downarrow 0,
\end{aligned}
$$

and hence (3.5) implies (3.7), as we desired to prove.

Proof of Proposition 3.1, Since $\partial \Omega$ is a real analytic hypersurface by Lemma 2.1, we can write

$$
\varphi\left(z_{1}, z_{2}\right)=\sum_{k=2}^{\infty} P_{k}\left(z_{1}, z_{2}\right) \text { for sufficiently small } \sqrt{z_{1}^{2}+z_{2}^{2}}
$$

where each $P_{k}\left(z_{1}, z_{2}\right)$ is a homogeneous polynomial of degree $k$ and in particular

$$
P_{2}\left(z_{1}, z_{2}\right)=-\frac{1}{2}\left(\kappa_{1}(\xi) z_{1}^{2}+\kappa_{2}(\xi) z_{2}^{2}\right)
$$

Now we compute the integrands of the expansion (3.7). For $P_{k}$ given in (3.11), we write

$$
P_{k}(v)=P_{k}(\cos \theta, \sin \theta) \text { for } v=(\cos \theta, \sin \theta) \text {. }
$$


By substituting this and (3.11) into (3.3), since

$$
\cos \eta=1-\frac{1}{2} \eta^{2}+\frac{1}{24} \eta^{4}+O\left(\eta^{6}\right) \text { and } \sin \eta=\eta-\frac{1}{6} \eta^{3}+O\left(\eta^{5}\right)
$$

we see that

$$
(R+s)\left(1-\frac{1}{2} \eta^{2}+\frac{1}{24} \eta^{4}+O\left(\eta^{6}\right)\right)-R-\sum_{k=2}^{\infty}(R+s)^{k}\left(\eta-\frac{1}{6} \eta^{3}+O\left(\eta^{5}\right)\right)^{k} P_{k}(v)=0
$$

Then, with (3.6) $\left(\eta=b_{1} s^{\frac{1}{2}}+b_{2} s+b_{3} s^{\frac{3}{2}}+O\left(s^{2}\right)\right)$ in hand, we equate to zero the coefficients of $s, s^{\frac{3}{2}}$, and $s^{2}$. The coefficient of $s$ gives

$$
1-\frac{1}{2} R b_{1}^{2}-R^{2} b_{1}^{2} P_{2}(v)=0
$$

The coefficient of $s^{\frac{3}{2}}$ gives

$$
-R b_{1} b_{2}-2 R^{2} b_{1} b_{2} P_{2}(v)-R^{3} b_{1}^{3} P_{3}(v)=0 .
$$

The coefficient of $s^{2}$ gives

$$
\begin{aligned}
& -\frac{1}{2}\left\{b_{1}^{2}+R b_{2}^{2}+2 R b_{1} b_{3}\right\}+\frac{1}{24} R b_{1}^{4} \\
& -P_{2}(v)\left\{2 R b_{1}^{2}+R^{2} b_{2}^{2}+2 R^{2} b_{1} b_{3}-\frac{1}{3} R^{2} b_{1}^{4}\right\} \\
& -3 R^{3} b_{1}^{2} b_{2} P_{3}(v)-R^{4} b_{1}^{4} P_{4}(v)=0 .
\end{aligned}
$$

Now, set

$$
\sigma_{j}=1-R \kappa_{j}(\xi)>0 \text { for } j=1,2 .
$$

Notice that

$$
\sigma_{1} \sigma_{2}=c
$$

where $c>0$ is the positive number given by (2.2).

In view of (3.7), with the aid of (3.13) and (3.12), we obtain:

$$
\frac{1}{2} b_{1}^{2}=\frac{1}{R\left(1+2 R P_{2}(v)\right)}=\frac{1}{R\left(\sigma_{1} \cos ^{2} \theta+\sigma_{2} \sin ^{2} \theta\right)} .
$$

The coefficient of $s$ in (3.1) is thus easily computed from this formula, by using (3.17) and (A.1):

$$
\frac{1}{2} \int_{0}^{2 \pi} b_{1}^{2} d \theta=\frac{2 \pi}{R \sqrt{c}}
$$

here $c$ is the positive number given by (2.2) . 
By using (3.14) and (3.18), we have:

$$
b_{1} b_{2}=-\frac{2^{\frac{3}{2}} R^{\frac{1}{2}} P_{3}(v)}{\left(\sigma_{1} \cos ^{2} \theta+\sigma_{2} \sin ^{2} \theta\right)^{\frac{5}{2}}},
$$

and hence we get

$$
\int_{0}^{2 \pi} b_{1} b_{2} d \theta=0
$$

since $b_{1} b_{2}$ is the sum of odd functions of either $\cos \theta$ or $\sin \theta$ because of (3.20). Thus, by (3.7) the coefficient of $s^{3 / 2}$ in (3.1) is zero.

Finally, it follows from (3.15), (3.18), and (3.20) that

$$
\begin{aligned}
\frac{1}{2}\left(b_{2}^{2}+2 b_{1} b_{3}-\frac{1}{12} b_{1}^{4}\right)= & -\frac{7}{6 R^{2}\left(\sigma_{1} \cos ^{2} \theta+\sigma_{2} \sin ^{2} \theta\right)^{2}}+\frac{1}{6 R^{2}\left(\sigma_{1} \cos ^{2} \theta+\sigma_{2} \sin ^{2} \theta\right)^{3}} \\
& -\frac{4 P_{2}(v)}{R\left(\sigma_{1} \cos ^{2} \theta+\sigma_{2} \sin ^{2} \theta\right)^{2}}+\frac{4 P_{2}(v)}{3 R\left(\sigma_{1} \cos ^{2} \theta+\sigma_{2} \sin ^{2} \theta\right)^{3}} \\
& +\frac{12 R^{2}\left(P_{3}(v)\right)^{2}}{\left(\sigma_{1} \cos ^{2} \theta+\sigma_{2} \sin ^{2} \theta\right)^{4}}-\frac{4 R P_{4}(v)}{\left(\sigma_{1} \cos ^{2} \theta+\sigma_{2} \sin ^{2} \theta\right)^{3}} .
\end{aligned}
$$

A long but important computation, that is carried out in the Appendix (Lemmas A.3 and A.4), then yields the coefficient of $s^{2}$ in (3.1), that is

$$
\begin{aligned}
\frac{R^{2}}{2} \int_{0}^{2 \pi}\left(b_{2}^{2}+2 b_{1} b_{3}-\frac{1}{12} b_{1}^{4}\right) & d \theta=\frac{\pi}{8 c \sqrt{c}}\left\{\left(R^{2} K+c-1\right)^{2}-4 c(c+3)\right. \\
- & \left.\frac{4}{3} c R^{4}\left[\left(1-R \kappa_{1}\right)^{-3}\left(\varphi_{111}\right)^{2}+\left(1-R \kappa_{2}\right)^{-3}\left(\varphi_{222}\right)^{2}\right]\right\}
\end{aligned}
$$

where the derivatives of $\varphi$ are evaluated at $(0,0)$.

In the last formula, we set

$$
g=-\frac{4}{3} c R^{4}\left[\left(1-R \kappa_{1}\right)^{-3}\left(\varphi_{111}\right)^{2}+\left(1-R \kappa_{2}\right)^{-3}\left(\varphi_{222}\right)^{2}\right]
$$

that is clearly non-positive and is null if and only if both third derivatives vanish.

In the next proposition, $\kappa_{1}^{*}$ and $\kappa_{2}^{*}$ denote the principal curvatures of $\Gamma_{*}$ at the point $x^{*}=\xi+\rho_{*} \nu(\xi)$ defined by (2.16) with $\rho_{*}=R /(1+\sqrt{c}), K^{*}=\kappa_{1}^{*} \kappa_{2}^{*}$, and $g$ is the function appearing in (3.1), whose expression is given by (3.23).

Proposition 3.3 It holds that

$$
\left(\kappa_{2}^{*}-\kappa_{1}^{*}\right)^{2} g=-\frac{4 R^{4}}{3 \sqrt{c}} \cdot \frac{\left\|\nabla K^{*}\right\|^{2}}{\left(1+\rho_{*} \kappa_{1}^{*}\right)^{3}\left(1+\rho_{*} \kappa_{2}^{*}\right)^{3}}
$$


or, in terms of the invariants $H^{*}$ and $K^{*}$,

$$
\left\|\nabla K^{*}\right\|^{2}=\frac{3 \sqrt{c}}{R^{4}} g\left[K^{*}-\left(H^{*}\right)^{2}\right]\left[1+2 \rho_{*} H^{*}+\rho_{*}^{2} K^{*}\right]^{3},
$$

where $\left\|\nabla K^{*}\right\|$ is the length of the gradient of the Gauss curvature $K^{*}$ with respect to the induced metric of the hypersurface $\Gamma_{*}$.

Proof. Note that

$$
\kappa_{j}=\frac{\kappa_{j}^{*}}{1+\rho_{*} \kappa_{j}^{*}} \text { for } j=1,2, \quad \kappa_{1}^{*}+\kappa_{2}^{*}=\frac{1-c}{R \sqrt{c}} \text { and } \rho_{*}=\frac{R}{1+\sqrt{c}} .
$$

First notice that formula (3.24) holds true if $\kappa_{1}=\kappa_{2}$ or, which is equivalent, if $\kappa_{1}^{*}=\kappa_{2}^{*}$. In fact, in this case, the Gauss curvature $K^{*}$ of $\Gamma_{*}$ attains its maximum value $\left(H^{*}\right)^{2}$ (at $x^{*}$ ). This means that $\nabla K^{*}$ vanishes $\left(\right.$ at $\left.x^{*}\right)$ and hence both sides of (3.24) equal zero.

We now suppose that $\kappa_{1} \neq \kappa_{2}$. Thus, by using the Monge principal coordinate system ([BL, p. 156]), we have as $\sqrt{z_{1}^{2}+z_{2}^{2}} \rightarrow 0$ that

$$
\begin{aligned}
\varphi\left(z_{1}, z_{2}\right)= & -\frac{1}{2} \kappa_{1} z_{1}^{2}-\frac{1}{2} \kappa_{2} z_{2}^{2} \\
& -\frac{1}{6}\left\{\frac{\partial \kappa_{1}}{\partial z_{1}} z_{1}^{3}+3 \frac{\partial \kappa_{1}}{\partial z_{2}} z_{1}^{2} z_{2}+3 \frac{\partial \kappa_{2}}{\partial z_{1}} z_{1} z_{2}^{2}+\frac{\partial \kappa_{2}}{\partial z_{2}} z_{2}^{3}\right\}+O\left(\left(z_{1}^{2}+z_{2}^{2}\right)^{2}\right) .
\end{aligned}
$$

Therefore, we have at $(0,0)$ :

Hence, we obtain from (3.23):

$$
\varphi_{111}=-\frac{\partial \kappa_{1}}{\partial z_{1}} \text { and } \varphi_{222}=-\frac{\partial \kappa_{2}}{\partial z_{2}} .
$$

$$
-\frac{3 \sqrt{c} g}{4 R^{4}}\left(1+\rho_{*} \kappa_{1}^{*}\right)^{3}\left(1+\rho_{*} \kappa_{2}^{*}\right)^{3}=\left(1+\rho_{*} \kappa_{1}^{*}\right)^{2}\left(\frac{\partial \kappa_{1}^{*}}{\partial z_{1}}\right)^{2}+\left(1+\rho_{*} \kappa_{2}^{*}\right)^{2}\left(\frac{\partial \kappa_{1}^{*}}{\partial z_{2}}\right)^{2} .
$$

By recalling that $\Gamma^{*}$ is parameterized in $z$ by

$$
(z, \varphi(z))-\rho_{*} \frac{1}{\sqrt{1+\left|\nabla_{z} \varphi(z)\right|^{2}}}\left(-\nabla_{z} \varphi(z), 1\right)
$$

we have at $z=0$ (that is at $x^{*} \in \Gamma_{*}$ ) that

$$
\begin{aligned}
\left\|\nabla K^{*}\right\|^{2} & =\left(1-\rho_{*} \kappa_{1}\right)^{-2}\left(\frac{\partial K^{*}}{\partial z_{1}}\right)^{2}+\left(1-\rho_{*} \kappa_{2}\right)^{-2}\left(\frac{\partial K^{*}}{\partial z_{2}}\right)^{2} \\
& =\left(1+\rho_{*} \kappa_{1}^{*}\right)^{2}\left(\frac{\partial K^{*}}{\partial z_{1}}\right)^{2}+\left(1+\rho_{*} \kappa_{2}^{*}\right)^{2}\left(\frac{\partial K^{*}}{\partial z_{2}}\right)^{2} .
\end{aligned}
$$

Notice now that

$$
\left(\frac{\partial K^{*}}{\partial z_{j}}\right)^{2}=\left(\frac{\partial \kappa_{1}^{*}}{\partial z_{j}}\right)^{2}\left(\kappa_{2}^{*}-\kappa_{1}^{*}\right)^{2} \text { for } j=1,2,
$$

since $K^{*}=\kappa_{1}^{*} \kappa_{2}^{*}$ and $\kappa_{1}^{*}+\kappa_{2}^{*}=\frac{1-c}{R \sqrt{c}}$.

Therefore, combining these with (3.26) gives the formula (3.24), which completes the proof of Proposition 3.3 . 


\section{Classification of stationary isothermic surfaces in $\mathbb{R}^{3}$}

We present two proofs of Theorem 1.1, each one with its own interest.

Proof of Theorem 1.1. Based on Propositions 2.3, 3.1 and 3.3, this proof relies on the theories of properly embedded minimal surfaces and properly embedded constant mean curvature surfaces in $\mathbb{R}^{3}$ and the theory of transnormal functions and transnormal systems.

First of all, we note that, being parallel to $\partial \Omega$, both $\Gamma$ and $\Gamma_{*}$ are unbounded and connected, which are properties they inherit from $\partial \Omega$.

Since $\Omega$ is uniformly dense in $\Gamma$, by Proposition 3.1 , there exists a constant $d$ such that

$$
h(K)+g=d \text { on } \partial \Omega .
$$

Moreover, since

$$
H^{*}=\frac{1-c}{2 R \sqrt{c}}, \quad \rho_{*}=\frac{R}{1+\sqrt{c}}, \quad \text { and } \quad K=\frac{K^{*}}{1+2 \rho_{*} H^{*}+\rho_{*}^{2} K^{*}},
$$

after a few straightforward computations, Propositions 3.1 and 3.3 give that

$$
\left\|\nabla K^{*}\right\|^{2}=\Psi\left(K^{*}\right) \text { on } \Gamma_{*} \text { and } \Psi\left(\left(H^{*}\right)^{2}\right)=0,
$$

where $\Psi$ is a polynomial with coefficients depending only on $c, R$, and $d$, and the degree of $\Psi$ is at most 4 .

We distinguish two cases:
(A) $K^{*}$ is constant on $\Gamma_{*}$;
(B) $K^{*}$ is not constant on $\Gamma_{*}$.

In case $(\mathrm{A})$, since also $H^{*}$ is constant on $\Gamma_{*}$, then $\kappa_{1}^{*}$ and $\kappa_{2}^{*}$ are both constant on $\Gamma_{*}$ and hence $\Gamma_{*}$ must be either a plane or a circular cylinder, by a classical result. Thus, the conclusion of Theorem 1.1 holds true, since both $\partial \Omega$ and $\Gamma$ are parallel to $\Gamma_{*}$.

In case (B), the first equation in (4.2) shows that the Gauss curvature $K^{*}$ is a transnormal function on the connected complete Riemannian manifold $\Gamma_{*}$ and it induces a transnormal system $\mathcal{F}$ (see Wang [W], Miyaoka [Mi], and Bolton [B]). To be precise, in our case, each component of the level sets of $K^{*}$ is called either "a foil" or "a singular foil" if its dimension is either 1 or 0 respectively, and all the components of the level sets of $K^{*}$ generate $\mathcal{F}$. All the foils are parallel to each other and any geodesic normal to a foil is orthogonal to every foil. Here, every foil must be a regular curve properly embedded in $\Gamma_{*}$, and every singular foil must be a point in $\Gamma_{*}$ which is a component of the focal varieties (possibly empty) $V_{+}=\left\{x \in \Gamma_{*}: K^{*}(x)=\max K^{*}\right\}$ and $V_{-}=\left\{x \in \Gamma_{*}: K^{*}(x)=\min K^{*}\right\}$. 
Since a regular curve properly embedded in $\Gamma_{*}$ is either a closed curve or a curve with infinite length and $\Gamma_{*}$ is unbounded, we have that

$$
\Gamma_{*} \text { is homeomorphic to either } \mathbb{S}^{1} \times \mathbb{R} \text { or } \mathbb{R}^{2} \text {. }
$$

This result was proved by Miyaoka in [Mi, Theorem 1.1]. For instance, if there exists a singular foil, then every foil in a neighborhood of it must be a closed curve and eventually $\Gamma_{*}$ must be homeomorphic to $\mathbb{R}^{2}$, and if there is no singular foil and one foil is a closed curve, then $\Gamma_{*}$ must be homeomorphic to $\mathbb{S}^{1} \times \mathbb{R}$.

Accordingly, it suffices to prove that (B) contradicts the fact that $\Gamma_{*}$ has constant mean curvature, as guaranteed by Proposition 2.3 . We arrive at this conclusion by examining two possibilities.

(I) If $\Gamma_{*}$ has non-zero constant mean curvature (that is when the constant $c$ in (2.2) is different from 1), Proposition 2.3, together with (4.3), shows that $\Gamma_{*}$ is properly embedded and of finite topology in $\mathbb{R}^{3}$, it is homeomorphic to either $\mathbb{S}^{2} \backslash\{\mathcal{N}, \mathcal{S}\}$ or $\mathbb{S}^{2} \backslash\{\mathcal{N}\}$ (here, $\mathcal{N}$ and $\mathcal{S}$ denote the north and south poles of the sphere $\mathbb{S}^{2}$ ), and each of its ends corresponds to each pole. Then, a theorem due to Meeks [Me, Theorem 1, p.540] shows that $\Gamma_{*}$ is homeomorphic to $\mathbb{S}^{2} \backslash\{\mathcal{N}, \mathcal{S}\}$ and, moreover, a theorem due to Korevaar-Kusner-Solomon [KoKS, Theorem 2.11, p. 476] shows that $\Gamma_{*}$ must be either a circular cylinder or an unduloid. See also Kenmotsu [Ke, p. 46] for an unduloid and $[\mathrm{KoK}]$ for a survey of properly embedded surfaces in $\mathbb{R}^{3}$ with constant mean curvature.

Since $K^{*}$ is not constant on $\Gamma_{*}$ by assumption (B), we have that $\Gamma_{*}$ is an unduloid, and hence $\partial \Omega$ is parallel to an unduloid, by Proposition 2.3. Thus, we can choose two points $P, Q \in \partial \Omega$ such that

$$
K(P)=K_{+}>0 \text { and } K(Q)=K_{-}<0
$$

$P$ and $Q$ lie on $\partial \Omega$ at the maximum (minimum) distance from the common axis of $\partial \Omega$ and $\Gamma_{*}$. The symmetry of $\partial \Omega$ ensures that the function $g$ in Proposition 3.1 vanishes at $P$ and $Q$, and hence we obtain that

$$
h(K(P))+g=h\left(K_{+}\right) \text {and } h(K(Q))+g=h\left(K_{-}\right)
$$

On the other hand, by the intermediate value theorem, there are points $P_{*}^{ \pm}$in $\partial \Omega$ with $0<K\left(P_{*}^{+}\right)<K_{+}$and $K_{-}<K\left(P_{*}^{-}\right)<0$. Since $h(K)=R^{4} K^{2}+2(c-1) R^{2} K+h(0)$ and $g \leq 0$, we obtain that $h\left(K\left(P_{*}^{+}\right)\right)+g \leq h\left(K\left(P_{*}^{+}\right)\right)<h\left(K_{+}\right)$, if $c>1$, and $h\left(K\left(P_{*}^{-}\right)\right)+g \leq$ $h\left(K\left(P_{*}^{-}\right)\right)<h\left(K_{-}\right)$, if $0<c<1$. These contradict (4.5) because of (4.1). 
(II) If $\Gamma_{*}$ has zero mean curvature (that is when $c=1$ ), again we can claim that $\Gamma_{*}$ is a properly embedded and of finite topology in $\mathbb{R}^{3}$ and that it is homeomorphic to either $\mathbb{S}^{2} \backslash\{\mathcal{N}, \mathcal{S}\}$ or $\mathbb{S}^{2} \backslash\{\mathcal{N}\}$ with each of its ends corresponding to each pole.

Thus, if $\Gamma_{*}$ is homeomorphic to $\mathbb{S}^{2} \backslash\{\mathcal{N}, \mathcal{S}\}$, either by combining results of Schoen [Sc] and Collin [C, Theorem 2, p. 2] or by combining results of López and Ros [LR] and Collin C, Theorem 2, p. 2] we get that $\Gamma_{*}$ must be a catenoid. Instead, if $\Gamma_{*}$ is homeomorphic to $\mathbb{S}^{2} \backslash\{\mathcal{N}\}$, a theorem of Meeks III and Rosenberg [MeR, Theorem 0.1, p. 728] implies that $\Gamma_{*}$ must be either a plane or a helicoid. See also [MeP] and [CM2] for a survey on the minimal surface theory in $\mathbb{R}^{3}$. Thus, since $K^{*}$ is not constant, $\Gamma_{*}$ must be either a catenoid or a helicoid.

Now, recall that for $c=1$ we have that

$$
K=\frac{K^{*}}{1+\rho_{*}^{2} K^{*}} .
$$

Assume that $\Gamma_{*}$ is a catenoid; then we know that $K^{*} \leq 0$ and $\kappa_{j}^{*} \rightarrow 0$ as $|x| \rightarrow \infty$ for $j=1,2$, and hence, by (4.6), we infer that

$$
K \leq 0 \text { and } \kappa_{j} \rightarrow 0 \text { as }|x| \rightarrow \infty(j=1,2) .
$$

Then, with the aid of the interior estimates for the minimal surface equation ( $\mathrm{GT}$, Corollary 16.7, p. 407]) and Schauder's interior estimates for higher order derivatives ([GT, Problem 6.1. (a), p. 141]), by proceeding as in [MaPS, Proof of Theorem 4.1, pp. 4833-4834], we see that, for any $k \in \mathbb{N}$, the $k$-th order derivatives of the function $\varphi$ in Proposition 3.1 converge to zero as $|x| \rightarrow \infty$; thus, it follows that

$$
h(K)+g \rightarrow h(0) \text { as }|x| \rightarrow \infty .
$$

On the other hand, since $\partial \Omega$ is parallel to the catenoid $\Gamma_{*}$, we choose a point $P_{0} \in \partial \Omega$, which is one of the points nearest to the common axis of $\partial \Omega$ and $\Gamma_{*}$, and which satisfies $K\left(P_{0}\right)=\inf _{P \in \partial \Omega} K(P)<0$. Again, the symmetry of $\partial \Omega$ ensures that the function $g$ in Proposition 3.1 vanishes at $P_{0}$ and we conclude that

$$
h\left(K\left(P_{0}\right)\right)+g=h\left(K\left(P_{0}\right)\right)=R^{4} K\left(P_{0}\right)^{2}+h(0)>h(0),
$$

that contradicts (4.7) because of (4.1).

Assume now that $\Gamma_{*}$ is a helicoid. Note that $K^{*}$ attains its negative minimum on the axis $\ell$ of $\Gamma_{*}$ and $K^{*}$ together with the principal curvatures $\kappa_{1}^{*}, \kappa_{2}^{*}$ tend to zero as the point goes away from $\ell$, as shown in [MoR, Example 3.46 (Helicoid), p. 91]. The same example 
and (4.6) imply that $K$ attains its negative minimum on the helix $\tilde{\ell}$ in $\partial \Omega$ corresponding to $\ell$, and $K$ together with the principal curvatures $\kappa_{1}, \kappa_{2}$ tend to zero as the point goes away from $\tilde{\ell}$. Therefore, by the same argument used in the case of the catenoid, as the point on $\partial \Omega$ goes away from $\tilde{\ell}$, we obtain that

$$
h(K)+g \rightarrow h(0)+0=h(0) .
$$

On the other hand, if we choose a point $\xi_{0} \in \tilde{\ell}$ corresponding to a point $x_{0}^{*} \in \ell$, since $K^{*}$ attains its negative minimum on $\ell$, at $x_{0}^{*}$ we have:

$$
\nabla K^{*}=0 \text { and } \kappa_{1}^{*} \neq \kappa_{2}^{*}
$$

this, together with (3.24), yields that $g=0$ at $\xi_{0} \in \tilde{\ell}$. Therefore, it follows that

$$
h\left(K\left(\xi_{0}\right)\right)+g=R^{4}(\min K)^{2}+h(0)+0>h(0),
$$

that contradicts (4.8). The proof is complete.

The proof of Theorem 1.1 presented above is divided in two steps. First, by using the transnormal condition (4.2) and the theories of CMC and minimal surfaces, we proved that either $\Gamma_{*}$ has constant Gauss curvature or it is globally isometric to an unduloid, a catenoid or a helicoid. Second, using the symmetries of the unduloid, the catenoid and the helicoid, and appropriate Schauder estimates (see the proof between Eqs. (4.6) and (4.7)), we showed that $\Gamma_{*}$ cannot be isometric to any of these surfaces. This second step of the proof makes use of general arguments that may be useful in other contexts, but we would like to remark that there is an elementary proof using the explicit expressions of the unduloid, catenoid and helicoid.

Alternative proof of Theorem 1.1. The proof proceeds by inspection, we just check that the transnormal condition (4.2), which is coordinate independent, does not hold on these surfaces.

(1) The unduloid. The family of the unduloids can parametrized using coordinates $(u, v) \in$ $\mathbb{R} /(2 \pi \mathbb{Z}) \times \mathbb{R}$, and two real parameters $b>a>0$, see e.g. [HMO]. In these coordinates the induced metric reads as

$$
\mathfrak{g}=\frac{1}{2}\left(a^{2}+b^{2}+\left(b^{2}-a^{2}\right) \sin \frac{2 v}{a+b}\right) d u^{2}+d v^{2},
$$

and the Gauss curvature is

$$
K=\frac{1}{(a+b)^{2}}-\frac{4 a^{2} b^{2}}{(a+b)^{2}}\left(a^{2}+b^{2}+\left(b^{2}-a^{2}\right) \sin \frac{2 v}{a+b}\right)^{-2} .
$$


A straightforward computation using the metric $\mathfrak{g}$ and the curvature $K$ yields that

$$
\|\nabla K\|^{2}=\left(K_{v}\right)^{2}=\left[1-(a+b)^{2} K\right]^{2}\left\{A_{1}+A_{2}\left[1-(a+b)^{2} K\right]^{1 / 2}+A_{3}\left[1-(a+b)^{2} K\right]\right\},
$$

where $A_{1}, A_{2}, A_{3}$ are real constants that can be written explicitly in terms of $a$ and $b$, but whose expressions are not relevant for our purposes. It can be checked that $A_{2} \neq 0$ for any values of $a$ and $b$, thus implying that $\|\nabla K\|^{2}$ is not a polynomial of $K$, and hence the surface $\Gamma_{*}$ cannot be an unduloid on account of Eq. (4.2).

(2) The catenoid. The family of the catenoids can be parametrized using coordinates $(u, v) \in \mathbb{R} /(2 \pi \mathbb{Z}) \times \mathbb{R}$ and a real constant $a>0$, cf. [MeP] and [CM2]. In this coordinate system the induced metric and Gauss curvature are

$$
\mathfrak{g}=a^{2} \cosh ^{2}\left(\frac{v}{a}\right) d u^{2}+\cosh ^{2}\left(\frac{v}{a}\right) d v^{2}, \quad K=\frac{-1}{a^{2} \cosh ^{4}(v / a)} .
$$

As before, after some computations the quantity $\|\nabla K\|^{2}$ can be written in terms of $K$ as

$$
\|\nabla K\|^{2}=\frac{\left(K_{v}\right)^{2}}{\cosh ^{2}(v / a)}=\frac{16}{a}(-K)^{5 / 2}+16 K^{3},
$$

which is not a polynomial of $K$. Therefore, $\Gamma_{*}$ cannot be a catenoid.

(3) The helicoid. The family of the helicoids can be parametrized with coordinates $(u, v) \in$ $\mathbb{R}^{2}$ and a real constant $a>0$, see $[\mathrm{MoR}]$. The induced metric and Gauss curvature read in these coordinates as

$$
\mathfrak{g}=\left(a^{2}+v^{2}\right) d u^{2}+d v^{2}, \quad K=\frac{-a^{2}}{\left(a^{2}+v^{2}\right)^{2}} .
$$

Algebraic calculations again yield that $\|\nabla K\|^{2}$ is not a polynomial of $K$, in fact,

$$
\|\nabla K\|^{2}=\left(K_{v}\right)^{2}=\frac{16}{a}(-K)^{5 / 2}+16 K^{3} .
$$

Therefore, $\Gamma_{*}$ cannot be a helicoid either.

Remark 4.1 We can also show that, in the proof of Theorem 1.1, if $K^{*}$ is not constant, then it is an isoparametric function, namely it satisfies the system of equations

$$
\left\|\nabla K^{*}\right\|^{2}=\Psi\left(K^{*}\right) \text { and } \Delta_{\Gamma_{*}} K^{*}=\Phi\left(K^{*}\right) \text { on } \Gamma_{*}
$$

for some continuous function $\Phi$; here, $\Delta_{\Gamma_{*}}$ is the Laplace-Beltrami operator on $\Gamma_{*}$. In our case, $\Phi$ and $\Psi$ are polynomials. 
In fact, the umbilical points of the surface $\Gamma_{*}$ of constant mean curvature are isolated (see [Ke, Proposition 1.4 and (1.40), p. 21] ), and by [Ke, (1.41), p. 22]

$$
\Delta_{\Gamma_{*}} \log \sqrt{\left(H^{*}\right)^{2}-K^{*}}-2 K^{*}=0 \text { on } \Gamma_{*} \backslash\{\text { umbilical points }\} .
$$

Therefore, it follows from the first equation of (4.2) that

$$
\Delta_{\Gamma_{*}} K^{*}=-4 K^{*}\left[\left(H^{*}\right)^{2}-K^{*}\right]-\frac{1}{\left(H^{*}\right)^{2}-K^{*}} \Psi\left(K^{*}\right) .
$$

Thus, the second equality of (4.2) guarantees that the right-hand side of this equation is written as $\Phi\left(K^{*}\right)$ for some polynomial $\Phi=\Phi(t)$ in $t \in \mathbb{R}$.

\section{Uniformly dense domains in $\mathbb{R}^{3}$ : the case $\Gamma=\partial \Omega$}

With the aid of Nitsche's result [N], the theory of embedded minimal surfaces of finite topology in $\left.\mathbb{R}^{3}([\mathrm{BB}], \mathrm{MeR}],[\mathrm{C}]\right)$ gives the following generalization of [MaPS, Theorem 1.4, p. 4824]:

Theorem 5.1 Let $S$ be a complete embedded minimal surface of finite topology in $\mathbb{R}^{3}$, and let $\Omega$ be one connected component of $\mathbb{R}^{3} \backslash S$.

If $\Omega$ is uniformly dense in $S(=\partial \Omega)$, then $S$ must be either a plane or a helicoid.

Proof. First of all, we note that $S$ must be properly embedded in $\mathbb{R}^{3}$ by Colding and Minicozzi II [CM1, Corollary 0.13, p. 214], and hence $S$ separates $\mathbb{R}^{3}$ into two connected components.

We shall use an argument similar to those used in MaPS, Proof of Theorem 1.4, p. 4833-4834]. Since $S$ is of finite topology, there exist a compact Riemann surface $M$ without boundary in $\mathbb{R}^{3}$ and a finite number of points $p_{1}, \ldots, p_{m} \in M$ such that $S$ is homeomorphic to $M \backslash\left\{p_{1}, \ldots, p_{m}\right\}$ and each end corresponds to each $p_{j}$. Then the structure theorem of Bernstein-Breiner [BB] (see also Meeks III-Rosenberg [MeR] and Collin [C]) shows the following:

(i) If $m \geq 2$, then $S$ has finite total curvature and each end of $S$ is asymptotic to either a plane or a half catenoid. See [BB, Corollary 1.4, p. 357] and [C, Theorem 2, p. 2];

(ii) If $m=1$, then either $S$ is a plane or it has infinite total curvature and its end is asymptotic to a helicoid. See [BB, Corollary 1.4, p. 357] and [MeR, Theorems 0.1 and 0.2, p. 728]; 
(iii) The Gauss curvature of $S$ is bounded, and hence the principal curvatures of $S$ are also bounded. See [Sc, Proposition 1, p. 801] and [HPR, Theorem 1, p. 1336] together with $[\mathrm{BB}]$ and $[\mathrm{MeR}]$.

See also $\left[\mathrm{MeP}\right.$ and $\mathrm{CM} 2$ for the minimal surface theory in $\mathbb{R}^{3}$.

Now, item (iii) above guarantees that there exists $\delta>0$ such that, for every $x \in S$, the connected component of $B_{\delta}(x) \cap S$ containing $x$ is written as a graph of a function over the tangent plane to $S$ at $x$ (see [CM2, Lemma 2.4, p. 74] for a proof). Hence combining the above (iii) with the interior estimates for the minimal surface equation (see GT, Corollary 16.7 , p. 407]) yields that the convergence in (i) and (ii) is in the $C^{k}$ local topology for any $k \in \mathbb{N}$.

Therefore, in view of the geometry of a hyperplane, a half catenoid, and a helicoid, each of (i) and (ii) gives a sequence of points $\left\{P_{j}\right\}$ in $S$ such that the principal curvatures of the connected component of $B_{\delta}\left(P_{j}\right) \cap S$ containing $P_{j}$ tend to zero uniformly as $j \rightarrow \infty$. Thus we can apply [MaPS, Theorem 4.1, p. 4833], which uses Nitsche's result [N], to complete the proof of Theorem 5.1 .

In terms of stationary isothermic surfaces, and using [MaPS, Theorems 1.1 and 1.3], Theorem 5.1 implies the following corollary:

Corollary 5.2 Let $\Omega$ be a domain in $\mathbb{R}^{3}$ whose boundary $\partial \Omega$ is an unbounded complete embedded surface. Assume that $\partial \Omega$ has finite topology and is a stationary isothermic surface of the solution $u$ of the Cauchy problem (1.1). Then $\partial \Omega$ must be a plane, a circular cylinder or a helicoid.

\section{Appendix}

The following list of definite integrals will be used in the calculations of Lemmas A.1 A.4. They easily follow by means of successive differentiations and algebraic manipulations of the formula:

$$
\frac{1}{2 \pi} \int_{0}^{2 \pi} \frac{d \theta}{\sigma_{1} \cos ^{2} \theta+\sigma_{2} \sin ^{2} \theta}=\sigma_{1}^{-\frac{1}{2}} \sigma_{2}^{-\frac{1}{2}}
$$

here, $\sigma_{1}$ and $\sigma_{2}$ are two positive parameters. For $0 \leq j \leq m$ and $m=0,1, \ldots$ we have:

$$
\frac{1}{2 \pi} \int_{0}^{2 \pi} \frac{(\cos \theta)^{2 j}(\sin \theta)^{2 m-2 j}}{\left(\sigma_{1} \cos ^{2} \theta+\sigma_{2} \sin ^{2} \theta\right)^{m+1}} d \theta=\frac{1}{2^{2 m}} \frac{(2 j) !(2 m-2 j) !}{m ! j !(m-j) !} \sigma_{1}^{-\frac{1}{2}-j} \sigma_{2}^{-\frac{1}{2}-(m-j)}
$$

for $0 \leq j \leq m$ and $m=0,1, \ldots$;

$$
\frac{1}{2 \pi} \int_{0}^{2 \pi} \frac{d \theta}{\left(\sigma_{1} \cos ^{2} \theta+\sigma_{2} \sin ^{2} \theta\right)^{m+1}}=\frac{1}{2^{2 m}} \sum_{j=0}^{m}\left(\begin{array}{c}
2 j \\
j
\end{array}\right)\left(\begin{array}{c}
2(m-j) \\
m-j
\end{array}\right) \sigma_{1}^{-\frac{1}{2}-j} \sigma_{2}^{-\frac{1}{2}-(m-j)} ;
$$




$$
\begin{aligned}
& \frac{1}{2 \pi} \int_{0}^{2 \pi} \frac{\cos ^{2} \theta d \theta}{\left(\sigma_{1} \cos ^{2} \theta+\sigma_{2} \sin ^{2} \theta\right)^{m+2}}= \\
& \frac{1}{2^{2 m+1}} \sum_{j=0}^{m} \frac{2 j+1}{m+1}\left(\begin{array}{c}
2 j \\
j
\end{array}\right)\left(\begin{array}{c}
2(m-j) \\
m-j
\end{array}\right) \sigma_{1}^{-\frac{1}{2}-j-1} \sigma_{2}^{-\frac{1}{2}-(m-j)}
\end{aligned}
$$

and

$$
\begin{aligned}
\frac{1}{2 \pi} \int_{0}^{2 \pi} \frac{\sin ^{2} \theta d \theta}{\left(\sigma_{1} \cos ^{2} \theta+\sigma_{2} \sin ^{2} \theta\right)^{m+2}}= \\
\frac{1}{2^{2 m+1}} \sum_{j=0}^{m} \frac{2 m-2 j+1}{m+1}\left(\begin{array}{c}
2 j \\
j
\end{array}\right)\left(\begin{array}{c}
2(m-j) \\
m-j
\end{array}\right) \sigma_{1}^{-\frac{1}{2}-j} \sigma_{2}^{-\frac{1}{2}-(m-j+1)}
\end{aligned}
$$

In this paper we use (A.2) for $1 \leq m \leq 3$, (A.3) for $1 \leq m \leq 2$, (A.4) and (A.5) for $m=1$, respectively. In the sequel, we set $\kappa_{j}=\kappa_{j}(\xi)$, for $j=1,2$, and abbreviate the partial derivatives of $\varphi$ with respect to $z_{1}$ and $z_{2}$ by subscripts; whenever it is needed, we shall specify their arguments: the varying point $z=\left(z_{1}, z_{2}\right)$ or the origin $(0,0)$.

The following two lemmas are preparatory for Lemmas A.3 and A.4 below.

Lemma A.1 The following formulas hold:

$$
\begin{gathered}
-\frac{2 c^{\frac{3}{2}}}{\pi} \int_{0}^{2 \pi} \frac{P_{2}(v) d \theta}{\left(\sigma_{1} \cos ^{2} \theta+\sigma_{2} \sin ^{2} \theta\right)^{2}}=\kappa_{1} \sigma_{2}+\kappa_{2} \sigma_{1} \\
-\frac{8 c^{\frac{5}{2}}}{\pi} \int_{0}^{2 \pi} \frac{P_{2}(v) d \theta}{\left(\sigma_{1} \cos ^{2} \theta+\sigma_{2} \sin ^{2} \theta\right)^{3}}=\kappa_{1}\left(3 \sigma_{2}^{2}+\sigma_{1} \sigma_{2}\right)+\kappa_{2}\left(\sigma_{1} \sigma_{2}+3 \sigma_{1}^{2}\right) \\
\frac{2^{5} 3^{2} c^{\frac{7}{2}}}{\pi} \int_{0}^{2 \pi} \frac{\left[P_{3}(v)\right]^{2} d \theta}{\left(\sigma_{1} \cos ^{2} \theta+\sigma_{2} \sin ^{2} \theta\right)^{4}}= \\
5\left(\varphi_{111}\right)^{2} \sigma_{2}^{3}+9\left(\varphi_{112}\right)^{2} \sigma_{1} \sigma_{2}^{2}+9\left(\varphi_{122}\right)^{2} \sigma_{1}^{2} \sigma_{2}+5\left(\varphi_{222}\right)^{2} \sigma_{1}^{3}+ \\
\frac{2^{5} c^{\frac{5}{2}}}{\pi} \int_{0}^{2 \pi} \frac{P_{4}(v) d \theta}{\left(\sigma_{1} \cos ^{2} \theta+\sigma_{2} \sin ^{2} \theta\right)^{3}}=\left(\varphi_{1111}\right) \sigma_{2}^{2}+2\left(\varphi_{1122}\right) \sigma_{1}^{2} \sigma_{2}+6\left(\varphi_{112}\right)\left(\varphi_{222}\right) \sigma_{1}^{2} \sigma_{2} ;
\end{gathered}
$$

Here, we mean that the derivatives of $\varphi$ are evaluated at $(0,0)$.

Proof. Since $-2 P_{2}(v)=\kappa_{1} \cos ^{2} \theta+\kappa_{2} \sin ^{2} \theta$, with the aid of (3.17), the first formula follows from (A.2) for $m=1$, and the second one follows from (A.4) and (A.5) for $m=1$. 
Observe that

$$
\begin{aligned}
36\left[P_{3}(v)\right]^{2}=\{ & \left.\left(\cos \theta \partial_{1}+\sin \theta \partial_{2}\right)^{3} \varphi\right\}^{2} \\
=\left(\varphi_{111}\right)^{2} & \cos ^{6} \theta+9\left(\varphi_{112}\right)^{2} \cos ^{4} \theta \sin ^{2} \theta+9\left(\varphi_{122}\right)^{2} \cos ^{2} \theta \sin ^{4} \theta+\left(\varphi_{222}\right)^{2} \sin ^{6} \theta \\
+6\left(\varphi_{111}\right)\left(\varphi_{122}\right) & \cos ^{4} \theta \sin ^{2} \theta+6\left(\varphi_{112}\right)\left(\varphi_{222}\right) \cos ^{2} \theta \sin ^{4} \theta \\
& +[\text { the sum of odd functions of either } \cos \theta \text { or } \sin \theta],
\end{aligned}
$$

and

$$
\begin{aligned}
24 P_{4}(v)=\left(\cos \theta \partial_{1}+\sin \theta \partial_{2}\right)^{4} \varphi & \\
=\left(\varphi_{1111}\right) \cos ^{4} \theta & +6\left(\varphi_{1122}\right) \cos ^{2} \theta \sin ^{2} \theta+\left(\varphi_{2222}\right) \sin ^{4} \theta \\
& +[\text { the sum of odd functions of either } \cos \theta \text { or } \sin \theta] .
\end{aligned}
$$

Then, with the aid of (3.17), the third and fourth formulas follow from (A.2) with $m=3$ and $m=2$ respectively.

Lemma A.2 Let $\varphi$ be the function representing $\partial \Omega$ locally as in Proposition 3.1 . If $\Omega$ is uniformly dense in $\Gamma$, then

$$
\begin{gathered}
\sigma_{2} \varphi_{111}+\sigma_{1} \varphi_{122}=0, \quad \sigma_{1} \varphi_{222}+\sigma_{2} \varphi_{112}=0, \\
\sigma_{1}^{-1} \varphi_{1111}+\sigma_{2}^{-1} \varphi_{1122}=-\frac{2 R}{c}\left\{\varphi_{111} \varphi_{122}-\left(\varphi_{112}\right)^{2}\right\} \\
+\frac{1}{R c}\left[4(c-1) \kappa_{1}^{2}+R \kappa_{1}^{2}\left(\kappa_{1}+3 \kappa_{2}\right)\right],
\end{gathered}
$$

and

$$
\begin{aligned}
\sigma_{2}^{-1} \varphi_{2222}+\sigma_{1}^{-1} \varphi_{1122}=-\frac{2 R}{c}\left\{\varphi_{222} \varphi_{112}-\right. & \left.\left(\varphi_{122}\right)^{2}\right\} \\
& +\frac{1}{R c}\left[4(c-1) \kappa_{2}^{2}+R \kappa_{2}^{2}\left(\kappa_{2}+3 \kappa_{1}\right)\right] .
\end{aligned}
$$

Here, $\sigma_{1}$ and $\sigma_{2}$ are given by (3.16) and the derivatives of $\varphi$ are evaluated at $(0,0)$.

Proof. Since (2.2) gives

$$
-R\left(\kappa_{1}+\kappa_{2}\right)+R^{2} \kappa_{1} \kappa_{2}=c-1,
$$

the function $\varphi\left(z_{1}, z_{2}\right)$ satisfies the partial differential equation:

$$
\begin{gathered}
R \sqrt{1+\varphi_{1}^{2}+\varphi_{2}^{2}}\left\{\left(1+\varphi_{2}^{2}\right) \varphi_{11}-2 \varphi_{1} \varphi_{2} \varphi_{12}+\left(1+\varphi_{1}^{2}\right) \varphi_{22}\right\}+ \\
R^{2}\left\{\varphi_{11} \varphi_{22}-\left(\varphi_{12}\right)^{2}\right\}=(c-1)\left(1+\varphi_{1}^{2}+\varphi_{2}^{2}\right)^{2}
\end{gathered}
$$


for $z$ in a neighborhood of $(0,0)$.

Recall that at $(0,0)$

$$
\varphi_{1}=\varphi_{2}=\varphi_{12}=0 \text { and } \varphi_{j j}=-\kappa_{j}(\xi) \text { for } j=1,2 \text {. }
$$

By differentiating (A.10) with respect to $z_{1}$, we obtain

$$
\begin{aligned}
& R \frac{\varphi_{1} \varphi_{11}+\varphi_{2} \varphi_{12}}{\sqrt{1+\varphi_{1}^{2}+\varphi_{2}^{2}}}\left\{\left(1+\varphi_{2}^{2}\right) \varphi_{11}-2 \varphi_{1} \varphi_{2} \varphi_{12}+\left(1+\varphi_{1}^{2}\right) \varphi_{22}\right\} \\
& +R \sqrt{1+\varphi_{1}^{2}+\varphi_{2}^{2}}\left\{2 \varphi_{2} \varphi_{12} \varphi_{11}+\left(1+\varphi_{2}^{2}\right) \varphi_{111}-2 \varphi_{11} \varphi_{2} \varphi_{12}-2 \varphi_{1}\left(\varphi_{12}\right)^{2}-2 \varphi_{1} \varphi_{2} \varphi_{112}\right. \\
& \left.+2 \varphi_{1} \varphi_{11} \varphi_{22}+\left(1+\varphi_{1}^{2}\right) \varphi_{122}\right\} \\
& +R^{2}\left(\varphi_{111} \varphi_{22}+\varphi_{11} \varphi_{122}-2 \varphi_{12} \varphi_{112}\right) \\
& =4(c-1)\left(1+\varphi_{1}^{2}+\varphi_{2}^{2}\right)\left(\varphi_{1} \varphi_{11}+\varphi_{2} \varphi_{12}\right)
\end{aligned}
$$

Letting $z=(0,0)$ in $($ A.12 $)$ yields, in view of (A.11), that

$$
R\left(\varphi_{111}+\varphi_{122}\right)-R^{2}\left(\kappa_{2} \varphi_{111}+\kappa_{1} \varphi_{122}\right)=0
$$

and hence the first formula in (A.6). By differentiating (A.10) with respect to $z_{2}$, a similar calculation gives the second formula in (A.6).

Again, by differentiating (A.12) with respect to $z_{1}$, then letting $z=(0,0)$ in the resulting equation, and using (A.11), we get

$$
\begin{aligned}
-R \kappa_{1}^{2}\left(\kappa_{1}+\kappa_{2}\right)+ & R\left\{\varphi_{1111}-2 \kappa_{1}^{2} \kappa_{2}+\varphi_{1122}\right\} \\
& +R^{2}\left\{-\kappa_{2} \varphi_{1111}+2 \varphi_{111} \varphi_{122}-\kappa_{1} \varphi_{1122}-2\left(\varphi_{112}\right)^{2}\right\}=4(c-1) \kappa_{1}^{2}
\end{aligned}
$$

Hence, with the aid of (3.16) and (3.17), we obtain (A.7). By differentiating (A.10) twice with respect to $z_{2}$ and then letting $z=(0,0)$ in the resulting equation, similar calculations yield (A.8).

We now complete the computation of the coefficient of $s^{2}$ in (3.1); we must integrate over $[0,2 \pi]$ the function in (3.22).

In view of (3.16), (3.17), (A.9), we preliminarily note that

$$
\sigma_{1}+\sigma_{2}=1+c-R^{2} K \text { and } \kappa_{1}+\kappa_{2}=\frac{1-c}{R}+R K
$$

In the following lemma, we use (A.3) for $m=1,2$ and the first two formulas in Lemma A.1 by using also (A.13), (3.17) and some algebraic manipulations, we obtain the integrals of the first four terms in (3.22). 
Lemma A.3 The following formulas hold:

$$
\begin{gathered}
-\int_{0}^{2 \pi} \frac{7 d \theta}{6 R^{2}\left(\sigma_{1} \cos ^{2} \theta+\sigma_{2} \sin ^{2} \theta\right)^{2}}=\frac{7 \pi}{6 R^{2} c^{3 / 2}}\left(R^{2} K-1-c\right), \\
\int_{0}^{2 \pi} \frac{d \theta}{6 R^{2}\left(\sigma_{1} \cos ^{2} \theta+\sigma_{2} \sin ^{2} \theta\right)^{3}}=\frac{\pi}{24 R^{2} c^{5 / 2}}\left[3 R^{4} K^{2}-6(1+c) R^{2} K+3 c^{2}+2 c+3\right], \\
-\int_{0}^{2 \pi} \frac{4 P_{2}(v) d \theta}{R\left(\sigma_{1} \cos ^{2} \theta+\sigma_{2} \sin ^{2} \theta\right)^{2}}=-\frac{2 \pi}{R^{2} c^{3 / 2}}\left(R^{2} K+c-1\right), \\
\int_{0}^{2 \pi} \frac{4 P_{2}(v) d \theta}{3 R\left(\sigma_{1} \cos ^{2} \theta+\sigma_{2} \sin ^{2} \theta\right)^{3}}=-\frac{\pi}{6 R^{2} c^{5 / 2}}\left[3 R^{4} K^{2}-2(3+c) R^{2} K-\left(c^{2}+2 c-3\right)\right] .
\end{gathered}
$$

We finally obtain the integrals of the last two terms in (3.22) by the last two formulas in Lemma A.1, Lemma A.2 and similar algebraic manipulations.

Lemma A.4 The following formula holds:

$$
\begin{aligned}
\int_{0}^{2 \pi} \frac{12 R^{2}\left[P_{3}(v)\right]^{2} d \theta}{\left(\sigma_{1} \cos ^{2} \theta+\sigma_{2} \sin ^{2} \theta\right)^{4}}- & \int_{0}^{2 \pi} \frac{4 R P_{4}(v) d \theta}{\left(\sigma_{1} \cos ^{2} \theta+\sigma_{2} \sin ^{2} \theta\right)^{3}} \\
=-\frac{\pi R^{2}}{6 \sqrt{c}}[(1- & \left.\left.R \kappa_{1}\right)^{-3}\left(\varphi_{111}\right)^{2}+\left(1-R \kappa_{2}\right)^{-3}\left(\varphi_{222}\right)^{2}\right] \\
& +\frac{\pi}{8 R^{2} c^{5 / 2}}\left\{(c+3)\left[R^{4} K^{2}+2(c-1) R^{2} K\right]-3(c-1)^{3}\right\} .
\end{aligned}
$$

Acknowledgement. The authors would like to thank Professor Reiko Miyaoka for her interest in their work and some useful discussions. Also, the authors are grateful to the anonymous referees for giving invaluable suggestions to improve the paper.

\section{References}

[A] A. D. Alexandrov, A characteristic property of spheres, Ann. Mat. Pura Appl. (4) 58 (1962), 305-315.

[ABG] M. Amar, L.R. Berrone and R. Gianni, A non local quantitative characterization of ellipses leading to a solvable differential relation, J. Inequal. in Pure \& Appl. Math. 9 (2008), 14 pp.

[BB] J. Bernstein and C. Breiner, Conformal structure of minimal surfaces with finite topology, Comment. Math. Helv., 86 (2011), 353-381. 
[BL] W. Blaschke and K. Leichtweiß, Elementare Differentialgeometrie, SpringerVerlag, Berlin, Heidelberg, New York, 1973.

[B] J. Bolton, Transnormal systems, Q. J. Math., Oxford II Ser. 24 (1973), 385-395.

[CM1] T. H. Colding and W. P. Minicozzi II, The Calabi-Yau conjectures for embedded surfaces, Ann. of Math. 167 (2008), 211-243.

[CM2] T. H. Colding and W. P. Minicozzi II, A Course in Minimal Surfaces, Graduate Studies in Math. vol. 121, Amer. Math. Soc., 2011.

[C] P. P. Collin, Topologie et courbure des surfaces minimales proprement plongées de $\mathbb{R}^{3}$, Ann. of Math. 145 (1997), 1-31.

[GT] D. Gilbarg and N.S. Trudinger, Elliptic Partial Differential Equations of Second Order, (Second Edition.), Springer-Verlag, Berlin, Heidelberg, New York, Tokyo, 1983.

[HMO] M. Hadzhilazova, I.M. Mladenov, and J. Oprea, Unduloids and their geometry, Arch. Math. 43 (2007), 417-429.

[HPR] L. Hauswirth, J. Pérez, and P. Romon, Embedded minimal ends of finite type, Trans. Amer. Math. Soc. 353 (2001), 1335-1370.

[Ke] K. Kenmotsu, Surfaces with Constant Mean Curvature, Transl. Math. Monogr., vol. 221, Amer. Math. Soc., 2003.

$[\mathrm{KoK}] \quad$ N. J. Korevaar and R. Kusner, The structure of constant mean curvature embeddings in Euclidean three space, Proc. Symposia Pure Math., 54 Part 1 (1993), 291-297.

[KoKS] N. J. Korevaar, R. Kusner, and B. Solomon, The structure of complete embedded surfaces with constant mean curvature, J. Diff. Geom. 30 (1989), 465-503.

[LR] F. J. López and A. Ros, On embedded complete minimal surfaces of genus zero, J. Diff. Geom. 33 (1991), 293-300.

[MaPS] R. Magnanini, J. Prajapat, and S. Sakaguchi, Stationary isothermic surfaces and uniformly dense domains, Trans. Amer. Math. Soc. 358 (2006), 4821-4841. 
[MM1] R. Magnanini and M. Marini, Characterization of ellipses as uniformly dense sets with respect to a family of convex bodies, Ann. Mat. Pura Appl., published online DOI 10.1007/s10231-013-0334-x.

[MM2] R. Magnanini and M. Marini, Characterization of ellipsoids as $K$-dense sets, submitted (2013), preprint arXiv:1308.0817.

[MaS1] R. Magnanini and S. Sakaguchi, Matzoh ball soup: heat conductors with a stationary isothermic surface, Ann. of Math. 156 (2002), 941-956.

[MaS2] R. Magnanini and S. Sakaguchi, Interaction between nonlinear diffusion and geometry of domain, J. Diff. Eqs. 252 (2012), 236-257.

[MaS3] R. Magnanini and S. Sakaguchi, Matzoh ball soup revisited: the boundary regularity issue, Math. Meth. Appl. Sci. 36 (2013), 2023-2032.

[Me] W. H. Meeks III, The topology and geometry of embedded surfaces of constant mean curvature, J. Diff. Geom. 27 (1988), 539-552.

[MeP] W. H. Meeks III and J. Pérez, A Survey on Classical Minimal Surface Theory, Univ. Lecture Series vol. 60, Amer. Math. Soc., 2012.

[MeR] W. H. Meeks III and H. Rosenberg, The uniqueness of the helicoid, Ann. of Math. 161 (2005), 727-758.

[Mi] R. Miyaoka, Transnormal functions on a Riemannian manifold, Diff. Geom. Appl. 31 (2013), 130-139.

[MoR] S. Montiel and A. Ros, Curves and Surfaces, Graduate Studies in Math. vol. 69, Amer. Math. Soc., 2005.

[N] J. C. C. Nitsche, Characterizations of the mean curvature and a problem of G. Cimmino, Analysis 15 (1995), 233-245.

[Sc] R. Schoen, Uniqueness, symmetry, and embeddedness of minimal surfaces, J. Diff. Geom. 18 (1983), 791-809.

[Sa] S. Sakaguchi, Stationary level surfaces and Liouville-type theorems characterizing hyperplanes, in "Geometric Properties of Parabolic and Elliptic PDE's", Springer INdAM Series, Vol. 2, 2013, 269-282. 
[W] Q. M. Wang, Isoparametric functions on Riemannian manifolds, I, Math. Ann. 277 (1987), 639-646. 